\title{
Kinematics of coronal mass ejections between 2 and 30 solar radii
}

\section{What can be learned about forces governing the eruption?`}

\author{
B. Vršnak ${ }^{1}$, D. Ruždjak ${ }^{1}$, D. Sudar ${ }^{1}$, and N. Gopalswamy ${ }^{2}$ \\ ${ }^{1}$ Hvar Observatory, Faculty of Geodesy, Kačićeva 26, 10000 Zagreb, Croatia \\ e-mail: bvrsnak@geodet.geof.hr \\ 2 NASA Goddard Space Flight Center, Greenbelt, MD 20771, USA
}

Received 30 January 2004 / Accepted 21 April 2004

\begin{abstract}
Kinematics of more than 5000 coronal mass ejections (CMEs) measured in the distance range 2-30 solar radii is investigated. A distinct anticorrelation between the acceleration, $a$, and the velocity, $v$, is found. In the linear form, it can be represented as $a=-k_{1}\left(v-v_{0}\right)$, where $v_{0}=400 \mathrm{~km} \mathrm{~s}^{-1}$, i.e., most of CMEs faster than $400 \mathrm{~km} \mathrm{~s}^{-1}$ decelerate, whereas slower ones generally accelerate.

After grouping CMEs into the width and mean-distance bins, it was found that the slope $k_{1}$ depends on these two parameters: $k_{1}$ is smaller for CMEs of larger width and mean-distance. Furthermore, the obtained CME subsets show distinct quadratic-form correlations, of the form $a=-k_{2}\left(v-v_{0}\right)\left|v-v_{0}\right|$. The value of $k_{2}$ decreases with increasing distance and width, whereas $v_{0}$ increases with the distance and is systematically larger than the slow solar wind speed by $100-200 \mathrm{~km} \mathrm{~s}^{-1}$.

The acceleration-velocity relationship is interpreted as a consequence of the aerodynamic drag. The excess of $v_{0}$ over the solar wind speed is explained assuming that in a certain fraction of events the propelling force is still acting in the considered distance range. In most events the inferred propelling force acceleration at 10 solar radii ranges between $a_{\mathrm{L}}=0$ and $10 \mathrm{~m} \mathrm{~s}^{-2}$, being on average smaller at larger distances. However, there are also events that show $a_{\mathrm{L}}>50 \mathrm{~m} \mathrm{~s}^{-2}$, as well as events indicating $a_{\mathrm{L}}<0$. Implications for the interplanetary motion of CMEs are discussed, emphasizing the prediction of the 1 a.u. arrival time.
\end{abstract}

Key words. magnetohydrodynamics (MHD) - Sun: corona - Sun: coronal mass ejections (CMEs) Sun: solar-terrestrial relations - Sun: solar wind - interplanetary medium

\section{Introduction}

Coronal mass ejection (CME) is a process in which the free energy contained in the coronal non-potential magnetic field is released in the form of kinetic and potential energy of ejected plasma (Forbes 2000). In large eruptions the magnetic flux of some $10^{23} \mathrm{~Wb}$ is expelled into the interplanetary space at velocities of the order of $1000 \mathrm{~km} \mathrm{~s}^{-1}$, carrying along $10^{13} \mathrm{~kg}$ of coronal plasma (Gosling 1990; Webb et al. 1994).

The non-potential field of the erupting structure carries the electric current, $I$, providing the Lorentz force that drives the eruption. The free energy of any current-carrying system can most generally be expressed as $W=L I^{2}$, where $L$ is the inductance of the structure (Jackson 1975; Landau et al. 1984; for a detailed discussion of the MHD aspect see Chen \& Krall 2002).

The inductance depends on the size of the current-carrying structure, implying that during the eruption $L$ increases (see,

* Appendices A and B are only available in electronic form at http://www. edpsciences.org e.g., Anzer 1978; Vršnak 1990; Chen 1996, and references therein). On the other hand, $L$ is related to the magnetic flux $\Phi$ associated with the current, $\Phi=L I$. Since it can be taken approximately that the erupting magnetic flux $\Phi$ does not change much, this implies that the current has to decrease as the erupting structure enlarges, $I \propto L^{-1}$. Because of the decreasing current, the Lorentz force weakens, eventually ceasing at large heliocentric distances.

Note that the condition $\Phi \approx$ const. could be violated in a limited time interval due to reconnection below the erupting flux tube (e.g., Vršank 1990; Lin \& Forbes 2000; see also Wang et al. 2003), or by an injection of the poloidal flux from the subphotospheric layers (e.g., Chen 1996). The supply of the poloidal flux prolongs the acceleration phase of the eruption.

The free energy of the system $W=L I^{2} \propto L^{-1}$ decreases, and if only the Lorentz force and gravity are considered, it should be directly transformed into kinetic and potential energy. However, CMEs travel through the ambient coronal/interplanetary $(\mathrm{C} / \mathrm{IP})$ plasma, causing the emission of MHD waves and appearance of the "aerodynamic" drag. The 
drag can have complex characteristics depending on the orientation of the ambient magnetic field (Vandas et al. 1995, 1996; Cargill et al. 1996; Cargill \& Schmidt 2002), but still can be expressed as:

$a_{\mathrm{d}}=-\gamma_{2}(v-w)|v-w|$,

where $v$ is the speed of the plasmoid moving in the medium flowing at velocity $w$ (Cargill et al. 1996). The parameter $\gamma_{2}$ depends on the cross-section $(A)$ and the mass $(m)$ of the plasmoid, and on the density $\left(\rho_{\mathrm{o}}\right)$ of the ambient plasma $\left(\gamma_{2} \propto\right.$ $\left.A \rho_{\mathrm{o}} / m\right)$.

The interaction of CMEs and the solar wind plasma is under favourable conditions "visualized" by the dekameterto-kilometer wavelength type II radio bursts. Such bursts are excited at the C/IP shocks that are driven by CMEs (e.g., Gopalswamy et al. 2001a).

As the Lorentz force and gravity decrease, the drag could become a dominant force. Indeed, Gopalswamy et al. (2000) inferred that in the IP space the CMEs that are faster than the solar wind decelerate, whereas the slower ones are additionally accelerated by the wind. The net acceleration was found to be anticorrelated with the CME speed; the issue was elaborated in a subsequent paper (Goplaswamy et al. 2001b) where the projection effects were partly eliminated and different distances from the Sun were considered. On the other hand, Gopalswamy et al. (2001a) analysed the relationship between acceleration and speed for fast $\left(v>900 \mathrm{~km} \mathrm{~s}^{-1}\right)$ and wide $\left(\phi>60^{\circ}\right) \mathrm{CMEs}$ associated with type II bursts, and found that in the upper corona faster CMEs show stronger deceleration. A similar result was obtained by Vršnak (2001a) who in addition showed that the deceleration rate statistically depends not only on the speed of ejecta, but also on the height. An empirical scaling law was established, describing the decrease of the average drag with increasing radial distance, which provides a better prediction of the Sun-Earth transit time (Vršnak \& Gopalswamy 2002).

In the papers by Vršnak (2001a) and Gopalswamy et al. (2001b) the acceleration/speed relationship (hereinafter $a-v$ relationship) was studied on relatively small samples of events of specific characteristics. The former one embraced only fast events showing an exponential-like decay of speed, whereas the second one included only fast and wide CMEs associated with type II bursts. For this reason, Vršnak et al. (2003) and Ruždjak et al. (2004) extended the investigation of the $a-v$ relationship to a large sample of more than 5000 CMEs observed by the Large Angle and Spectrometric Coronagraph (LASCO) aboard the Solar and Heliospheric Observatory (Brueckner et al. 1995) in the period 1996-2001. Again, the data showed a distinct $a-v$ anticorrelation.

In this paper we perform a detailed analysis of the $a-v$ relationship utilizing the same data set as in the preliminary reports by Vršnak et al. (2003) and Ruždjak et al. (2004). The aim of the paper is two-fold. Firstly, it is an extension of previous statistical analyses of kinematics of large samples of CMEs (e.g. Hundhausen 1993; Hundhausen et al. 1994; Harrison 1995; St. Cyr et al. 1999, 2000; Moon et al. 2002; Gopalswamy et al. 2003, and references therein). Secondly, the obtained empirical relationships are used as an insight into the physical background of CME dynamics in the near-Sun IP space. Both of these aspects could be essential in advancing the prediction of the CME arrival time at the Earth (Gopalswamy 2002).

The paper is organized as follows: the data set, the data sampling, and the parameters used are described in Sect. 2. Supplementary information concerning the statistical properties of the data set are shown in Appendix A. In Sects. 3 and 4 we present the empirical results, leaving explanations regarding the procedure for Appendix B. The results are discussed and interpreted in Sect. 5 and summarized in Sect. 6.

\section{The data}

The following analysis is based on the CME distancetime measurements, $R(t)$, where $R=r / r_{\odot}$ is the planeof-sky heliocentric distance $r$ normalized with respect to the solar radius $r_{\odot}$. The measurements are provided in the LASCO CME catalogue (Yashiro and Michalek, http://cdaw.gsfc.nasa.gov/CME_list/). We utilize the data from 5012 events recorded in the $2<R<30$ range from 1996 to 2001 . From this sample we extracted two subsamples: one consists of events which had been measured in $n \geq 4$ instants, and the other with $n \geq 6$ (hereinafter $n 4$-set and n6-set, containing 4609 and 3697 events, respectively). Such a sampling was applied to check how the accuracy of the acceleration and velocity estimates affects the results. Demanding a still larger $n$ would certainly improve the accuracy of individual kinematic data, but it would lead to a systematic exclusion of the fastest CMEs due to the limited image acquisition rate of the LASCO instruments.

The basic parameters treated in the following are:

- $R_{\mathrm{b}}$ and $R_{\mathrm{e}}$ - the smallest and the largest distance at which the CME was measured,

- $\Delta R=R_{\mathrm{e}}-R_{\mathrm{b}}$ and $\Delta t=t_{\mathrm{e}}-t_{\mathrm{b}}-$ the corresponding distance and time intervals,

- $R_{\mathrm{m}}=\left(R_{\mathrm{b}}+R_{\mathrm{e}}\right) / 2-$ mean radial distance for a given CME,

- $v_{\mathrm{m}}$ - mean velocity obtained using the linear fit to the $R(t)$ data, and the corresponding error $M_{v}$,

- $a_{\mathrm{m}}$ - mean acceleration received from the 2 nd degree polynomial fit, and the corresponding error $M_{a}$,

- $v_{\mathrm{b}}$ and $v_{\mathrm{e}}$ - the velocities at $R_{\mathrm{b}}$ and $R_{\mathrm{e}}$ evaluated from the 2nd degree polynomial fit,

- $v_{3}$ and $v_{5}-\mathrm{CME}$ velocities at $R=3$ and $R=5$ evaluated from the 2nd degree polynomial fit,

- $\phi$ - angular width of CME, measured at the height beyond which it remains more or less constant.

Statistical properties of the data set are given in Appendix A, where we show the distribution of $R_{\mathrm{b}}, \Delta R, R_{\mathrm{m}}, \phi, v_{\mathrm{m}}$, and $a_{\mathrm{m}}$. There is a weak positive correlation between the parameters $R_{\mathrm{m}}, v_{\mathrm{m}}$, and $\phi$ (also shown in Appendix A), whereas the acceleration is not correlated with the mean-distance or width.

In the preliminary reports by Vršnak et al. (2003) and Ruždjak et al. (2004) it was shown that CME accelerations and velocities are anticorrelated, indicating that the aerodynamic drag plays an important role in the CME dynamics. Since the drag acceleration depends on the ambient solar wind speed and density, as well as on the CME dimensions and density, 
it would be ideal if all events were recorded in the same range of heights. However, mean distances are found in the range from $R_{\mathrm{m}}=3$ to 20 (see Fig. 10 in Appendix A). Therefore, it is instructive to consider the velocity "normalized" to a particular height so that the ambient wind speed and density are the same for all events. Since a vast majority of events were measured from $R_{\mathrm{b}} \approx 3$, we included in the analysis the velocity $v_{3}$ as a kind of "initial velocity" at a fixed (plane-of-sky) height. Other reasons for using $v_{3}$ are explained in more detail and discussed in Sect. 5.1 and Appendix B.

The velocity $v_{5}$ represents (statistically) the velocity at $R$ somewhere in between the "initial distance" $R=3$ and the average value of mean-distances, $\bar{R}_{\mathrm{m}}$, which amounts to $\bar{R}_{\mathrm{m}} \approx 9$ (see Appendix A). We also considered $v\left(R_{\mathrm{m}}\right)$ obtained from the 2nd degree polynomial fit, but since $v\left(R_{\mathrm{m}}\right) \approx v_{\mathrm{m}}$ the difference in the outcome for $v\left(R_{\mathrm{m}}\right)$ and $v_{\mathrm{m}}$ turned out to be statistically insignificant. Hereafter, $v_{3}, v_{5}, v_{\mathrm{m}}$, and $v\left(R_{\mathrm{m}}\right)$ are denoted as "velocity options".

An important consequence of employing $v_{3}$ is that the events with $a_{\mathrm{m}}>0$ for which the fitted $R(t)$ parabolas do not have an intercept with the $R=3$ level dropped out from the analysed sample. Similarly, considering $v_{5}$ one excludes the events that show a large deceleration and are observed only across a relatively small range of heights, so that the fitted $R(t)$ parabola has no intercept with $R=5$. Such a "silent elimination" additionally filters out unreliable/odd data. In this way we got four subsamples which we denote n4v3-subset, n6v3-subset, n4v5-subset, and n6v5-subset, containing 4463, 3581,4570 , and 3681 events, respectively.

\section{Raw $a-v$ relationship}

In Fig. 1 we present the general relationship between CME accelerations and velocities. The four presented graphs are chosen to show basic characteristics of the $a-v$ correlation, and various statistical aspects of the relationship. Different subsets are chosen to illustrate that the general outcome is qualitatively the same, whatever sampling and velocity options are employed.

Since it is impossible to transparently present several thousands of data points in a single graph, we first present in Fig. 1a the dependence $a_{\mathrm{m}}\left(v_{\mathrm{m}}\right)$ by showing a 2-dimensional distribution of data. An example of the "all-data" $a_{\mathrm{m}}\left(v_{\mathrm{m}}\right)$ graph, analogous to the $v_{3}\left(a_{\mathrm{m}}\right)$ graph that is shown in Fig. 1b, can be found in the paper by Moon et al. (2002), or Ruždjak et al. (2004).

The complete $n 4 v 3$-subset data are shown in the $v_{3}\left(a_{\mathrm{m}}\right)$ graph in Fig. 1b, and in Fig. 1c we show the corresponding bin-averaged values with the standard deviations (for mean errors $M=\sigma / \sqrt{N}$ see Vršnak et al. 2003). The fit in Fig. 1c is obtained by attributing equal weights to all bin-averaged values in order to eliminate the effect of data grouping. The quadratic fit of the form $a_{\mathrm{m}}=-k_{2}\left(v-v_{0}\right)\left|v-v_{0}\right|$ is illustrated in Fig. 1d, where the n6v3-subset is used.

Note that the $a(v)$ graphs are presented in the most convenient form, where the acceleration is expressed in $\mathrm{m} \mathrm{s}^{-2}$ and the velocity in $\mathrm{km} \mathrm{s}^{-1}$. So, the slope $k_{1}$ of the linear regression line in such a presentation has the unit $\left[\mathrm{km}^{-1} \mathrm{~m} \mathrm{~s}^{-1}\right]=$ $\left[10^{-3} \mathrm{~s}^{-1}\right]$, thus the units-adjusted slope has the value $\gamma_{1}=$ $10^{-3} k_{1}$. Analogously, the parameter $k_{2}$, which appears in the
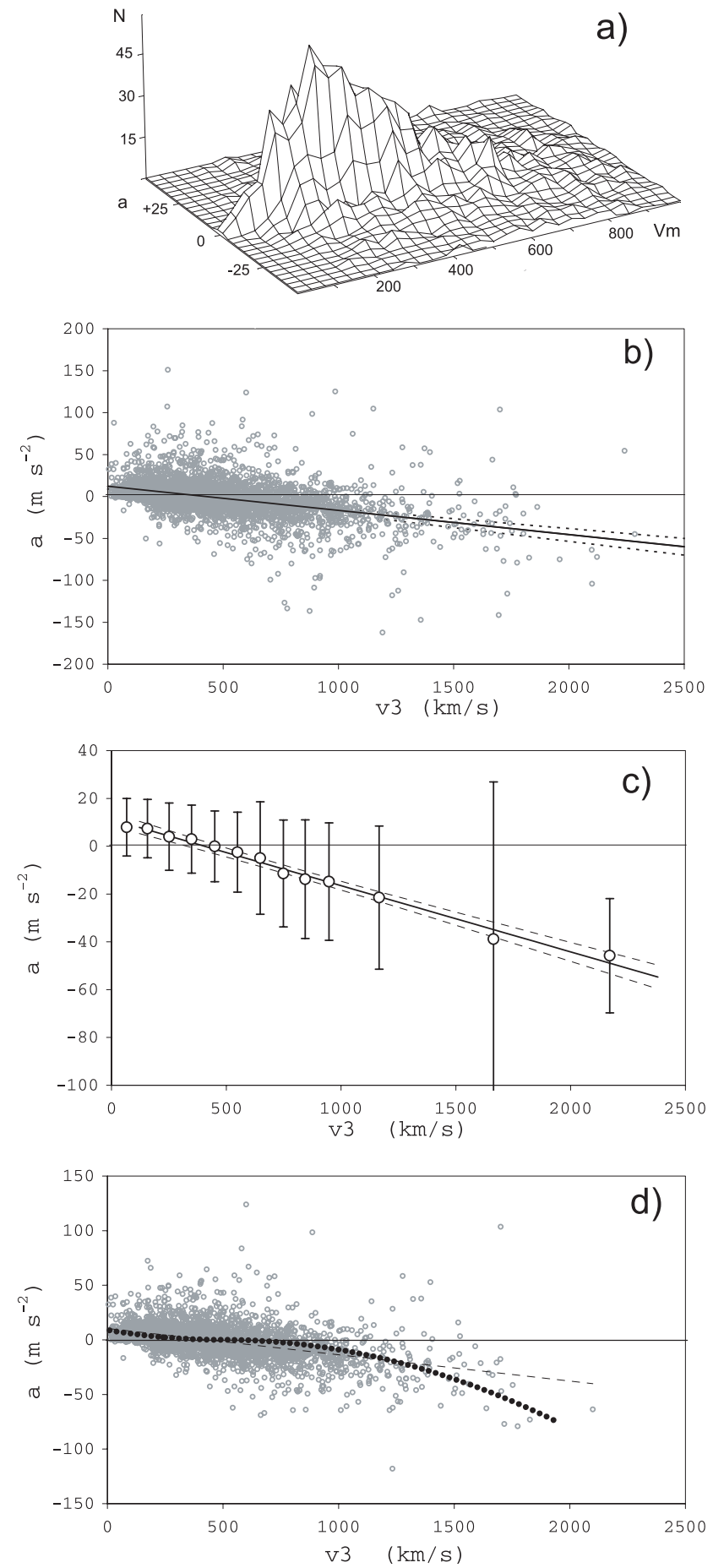

Fig. 1. The acceleration-speed correlation: a) 2D distribution of events in $a-v$ space for n $4 \mathrm{vm}$-subset. $N$ is the number of events in the $[\Delta a] \times$ $[\Delta v]=\left[5 \mathrm{~m} \mathrm{~s}^{-2}\right] \times\left[20 \mathrm{~km} \mathrm{~s}^{-1}\right]$ bins. b) The $\mathrm{n} 4 \mathrm{v} 3$ data. c) $\mathrm{n} 4 \mathrm{v} 3$ bin-averaged values $\bar{a}_{\mathrm{m}}$ versus $\bar{v}_{3}$ for $\Delta v=100 \mathrm{~km} \mathrm{~s}^{-1}$ bins (beyond $v>1000 \mathrm{~km} \mathrm{~s}^{-1}$, five successive bins are merged into $\Delta v=$ $500 \mathrm{~km} \mathrm{~s}^{-1}$ bins). Full lines in b) and c) show the linear least square fits, whereas $99 \%$ confidence lines are drawn dashed. d) Quadratic fit for n6v3 data (bold-dotted line) compared with the linear fit (thindashed line).

quadratic fit and has the unit $\left[\mathrm{m} \mathrm{km}^{-2}\right]=\left[10^{-3} \mathrm{~km}^{-1}\right]$, is related to the unit adjusted slope as $\gamma_{2}=10^{-3} k_{2}$, where $\gamma_{2}$ is expressed in $\mathrm{km}^{-1}$. 
Table 1. Summary of linear fits $a=-\gamma_{1}\left(v-v_{0}\right)$.

\begin{tabular}{lcccc}
\hline \hline$a_{\mathrm{m}}\left(v_{i}\right)$ & $\begin{array}{c}\text { Data } \\
\text { set }\end{array}$ & $\begin{array}{c}\gamma_{1} \\
10^{-3} \mathrm{~s}^{-1}\end{array}$ & $\begin{array}{c}v_{0} \\
\mathrm{~km} \mathrm{~s}^{-1}\end{array}$ & $C$ \\
\hline \multicolumn{5}{c}{ complete sets } \\
$a_{\mathrm{m}}\left(v_{\mathrm{m}}\right)$ & $\mathrm{n} 4 \mathrm{vm}$ & $0.0106 \pm 0.0012$ & $403 \pm 28$ & 0.13 \\
$a_{\mathrm{m}}\left(v_{\mathrm{m}}\right)$ & $\mathrm{n} 6 \mathrm{vm}$ & $0.0101 \pm 0.0010$ & $445 \pm 18$ & 0.16 \\
$a_{\mathrm{m}}\left(v_{3}\right)$ & $\mathrm{n} 4 \mathrm{v} 3$ & $0.0288 \pm 0.0010$ & $421 \pm 10$ & 0.41 \\
$a_{\mathrm{m}}\left(v_{3}\right)$ & $\mathrm{n} 6 \mathrm{v} 3$ & $0.0241 \pm 0.0008$ & $431 \pm 7$ & 0.46 \\
$a_{\mathrm{m}}\left(v_{5}\right)$ & $\mathrm{n} 4 \mathrm{v} 5$ & $0.0193 \pm 0.0011$ & $445 \pm 9$ & 0.25 \\
$a_{\mathrm{m}}\left(v_{5}\right)$ & $\mathrm{n} 6 \mathrm{v} 5$ & $0.0196 \pm 0.0009$ & $455 \pm 8$ & 0.34 \\
\hline Mean & \multicolumn{5}{c}{$0.019 \pm 0.007$} & $430 \pm 20$ \\
\hline \multicolumn{5}{c}{ bin-averaged } \\
$a_{\mathrm{m}}\left(v_{\mathrm{m}}\right)$ & $\mathrm{n} 4 \mathrm{vm}$ & $0.0113 \pm 0.0016$ & $303 \pm 65$ & 0.90 \\
$a_{\mathrm{m}}\left(v_{\mathrm{m}}\right)$ & $\mathrm{n} 6 \mathrm{vm}$ & $0.0108 \pm 0.0016$ & $350 \pm 45$ & 0.92 \\
$a_{\mathrm{m}}\left(v_{3}\right)$ & $\mathrm{n} 4 \mathrm{v} 3$ & $0.0266 \pm 0.0007$ & $407 \pm 15$ & 0.96 \\
$a_{\mathrm{m}}\left(v_{3}\right)$ & $\mathrm{n} 6 \mathrm{v} 3$ & $0.0244 \pm 0.0009$ & $425 \pm 7$ & 0.99 \\
$a_{\mathrm{m}}\left(v_{5}\right)$ & $\mathrm{n} 4 \mathrm{v} 5$ & $0.0186 \pm 0.0033$ & $368 \pm 71$ & 0.89 \\
$a_{\mathrm{m}}\left(v_{5}\right)$ & $\mathrm{n} 6 \mathrm{v} 5$ & $0.0188 \pm 0.0025$ & $401 \pm 58$ & 0.93 \\
\hline Mean & \multicolumn{5}{c}{$0.018 \pm 0.007$} & $380 \pm 40$ \\
\hline
\end{tabular}

The same regression analysis as the one shown in Fig. 1 is performed for all other velocity options and data-subsets. The results are summarized in the upper part of Table 1, where in the first two columns the velocity option and the data-subset label are indicated. In the $3 \mathrm{rd}$, and 4 th column we present the unit-adjusted slope, $\gamma_{1}=10^{-3} k_{1} \mathrm{~s}^{-1}$, and the $x$-axis intercept, $v_{0} \equiv v_{a=0}$, of the linear least squares fit, $a=-\gamma_{1}\left(v-v_{0}\right)$. In the last column the correlation coefficients $C$ are presented. According to the F-test the statistical significance $P_{\%}$ is larger than $99 \%$ for all correlations listed in Table $1\left(P_{\%}^{*}=100-P_{\%}\right.$ gives the probability for $C=0$ ).

In order to check how much the accuracy of measurements influences the obtained results, we successively excluded the events with the acceleration-error $M_{a}$ larger than 30, 20, $10 \mathrm{~m} \mathrm{~s}^{-2}$. It was found that the results do not depend significantly on such a subsampling.

In the lower part of Table 1 we show the results obtained using the bin-averaged values of $\bar{a}$ and $\bar{v}$ (Fig. 1c). The linear least squares fits presented in Table 1 exclude the highest velocity bins, $1500<v<2000 \mathrm{~km} \mathrm{~s}^{-1}$ and $2000<v<$ $2500 \mathrm{~km} \mathrm{~s}^{-1}$, where only a small number of events is found (e.g., in the n6v3-subset these two bins contain only 21 and 1 event, respectively).

Figure 1 and Table 1 reveal a distinct anticorrelation of accelerations and speeds. The largest correlation coefficients are found for the n6v3-subset and n4v3-subset, which will be addressed in Sect. 5.1 and Appendix B.

Inspecting Table 1 , one finds that the slopes are scattered in the range $\gamma_{1} \approx 0.01-0.03 \times 10^{-3} \mathrm{~s}^{-1}$. The acceleration $a_{\mathrm{m}}=0$ is found between $v_{0} \approx 350$ and $450 \mathrm{~km} \mathrm{~s}^{-1}$ in most of the data subsets considered. The bin-averaged data give systematically lower values of $v_{0}$ than the complete data sets $\left(\bar{v}_{0}=380 \pm 40\right.$ and $\bar{v}_{0}=430 \pm 20 \mathrm{~km} \mathrm{~s}^{-1}$, respectively).
Table 2. Summary of quadratic fits $a=-\gamma_{2}\left(v-v_{0}\right)\left|v-v_{0}\right|$.

\begin{tabular}{cccc}
\hline \hline$a_{\mathrm{m}}\left(v_{i}\right)$ & $\begin{array}{c}\text { Data } \\
\text { set }\end{array}$ & $\begin{array}{c}\gamma_{2} \\
10^{-6} \mathrm{~km}^{-1}\end{array}$ & $\begin{array}{c}v_{0} \\
\mathrm{~km} \mathrm{~s}^{-1}\end{array}$ \\
\hline$a_{\mathrm{m}}\left(v_{\mathrm{m}}\right)$ & $\mathrm{n} 4 \mathrm{vm}$ & 0.0061 & 84 \\
$a_{\mathrm{m}}\left(v_{\mathrm{m}}\right)$ & $\mathrm{n} 6 \mathrm{vm}$ & 0.0067 & 171 \\
$a_{\mathrm{m}}\left(v_{3}\right)$ & $\mathrm{n} 4 \mathrm{v} 3$ & 0.0192 & 162 \\
$a_{\mathrm{m}}\left(v_{3}\right)$ & $\mathrm{n} 6 \mathrm{v} 3$ & 0.0359 & 500 \\
$a_{\mathrm{m}}\left(v_{5}\right)$ & $\mathrm{n} 4 \mathrm{v} 5$ & 0.0179 & 266 \\
$a_{\mathrm{m}}\left(v_{5}\right)$ & $\mathrm{n} 6 \mathrm{v} 5$ & 0.0281 & 445 \\
\hline
\end{tabular}

The results shown in Table 1 can be summarized in general terms as:

$a_{\left[\mathrm{m} \mathrm{s}^{-2}\right]}=-k_{1}\left(v-v_{0}\right)_{\left[\mathrm{km} \mathrm{s}^{-1}\right]}=-0.02_{ \pm 0.01}\left(v-400_{ \pm 50}\right)$,

where the acceleration $a$ is expressed in $\mathrm{m} \mathrm{s}^{-2}$ and the velocity $v$ in $\mathrm{km} \mathrm{s}^{-1}$. Such an anticorrelation of accelerations and speeds is indicative of the aerodynamic drag (Cargill et al. 1996; Vršnak 2001a; Cargill \& Schmidt 2002). Indeed, the velocity $v_{0} \equiv v_{a=0} \approx 400 \mathrm{~km} \mathrm{~s}^{-1}$ is relatively close to the typical solar wind speed in the considered distance range (Sheeley et al. 1997). So, roughly speaking, CMEs faster than the solar wind decelerate, whereas slower ones accelerate. However, note that $v_{0}$ is in fact somewhat larger than the values measured within the LASCO field-of-view (Sheeley et al. 1997); the issue is addressed in Sect. 5.3.

Finally, we emphasize that Figs. 1a, b, and d expose a void of data-points in the range $v<150 \mathrm{~km} \mathrm{~s}^{-1}, a<0$, revealing that there are practically no slow events that decelerate.

Results presented by Gopalswamy et al. (2001b) and Vršnak (2001a) indicate that the $a-v$ relationship might be better approximated by the quadratic dependence of the form defined by Eq. (1) ${ }^{1}$. The quadratic fit parameters, given in Table 2, show a much larger scatter than the linear-fit ones (to be discussed in Sect. 5.3). Statistically, the most reliable quadratic fit is obtained for the n6v3-subset which is shown in Fig. 1d. For this sample the least squares fit gives $\gamma_{2}=0.036 \times 10^{-6} \mathrm{~km}^{-1}$ and $v_{0}=500 \mathrm{~km} \mathrm{~s}^{-1}$. The values averaged over all considered data sets are $\bar{\gamma}_{2}=0.019 \pm 0.012 \times 10^{-6} \mathrm{~km}^{-1}$ and $\bar{v}_{0}=270 \pm$ $170 \mathrm{~km} \mathrm{~s}^{-1}$. A more detailed treatment of quadratic fits is presented in Sect. 4.2, where it will be shown that they become statistically relevant after the data are sorted into the width and mean-distance bins.

\section{Dependencies $\gamma(\phi)$ and $\gamma\left(R_{\mathrm{m}}\right)$}

The $a-v$ anticorrelation indicates that the motion of CMEs in the $R=2-30$ range is strongly influenced by the aerodynamic drag. If so, it can be expected that the CME width $\phi$ is an important parameter since the drag acceleration depends on the dimensions of the moving body. On the other hand, the drag

\footnotetext{
1 When Eq. (1) will be used to fit the data, i.e., to describe the statistical relationship between the measured mean-accelerations and velocities we will call it the $a-v$ correlation or quadratic $a-v$ fit; when used to describe the instantaneous drag acceleration we will call it the $a(v)$-curve or $a(v)$-trajectory.
} 

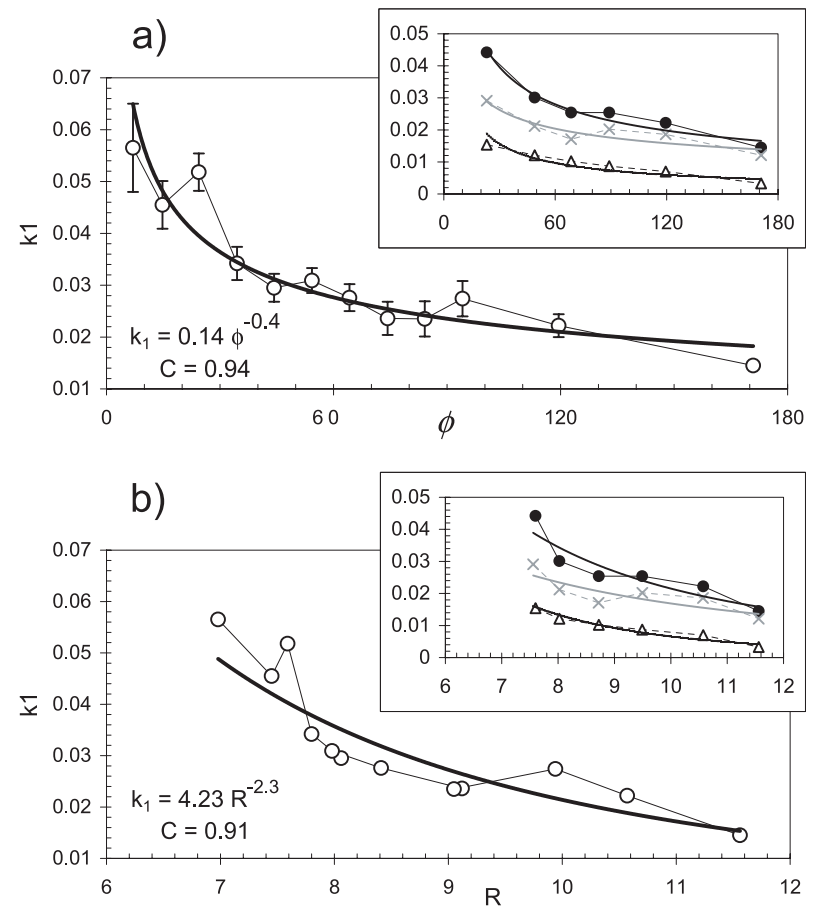

Fig. 2. Slopes $k_{1}=10^{3} \gamma_{1}$ of the $a(v)$ linear fits for the $n 4 \mathrm{v} 3$-subset data sorted into $\Delta \bar{\phi}=10^{\circ}$ bins: a) $k_{1}$ presented as a function of binaveraged widths $\bar{\phi}$; b) $k_{1}$ shown versus bin-averaged distances $\bar{R}_{\mathrm{m}}$. The power-law least squares fits are presented together with the correlation coefficients $C$. In the insets analogous results are presented for the $\Delta \phi=20^{\circ}$ bins using $\mathrm{n} 4 \mathrm{v} 3$-subset (dots), n4v5-subset (crosses), and $\mathrm{n} 4 \mathrm{vm}$-subset (triangles).

depends on the external density and flow speed, so one can suspect that another relevant parameter is the height range in which a particular CME is measured. To investigate the role of the parameters $\phi$ and $R_{\mathrm{m}}$ we rely mainly on the $\mathrm{n} 4$-set, which is large enough to provide a further sub-grouping of the data into a number of $\phi$ and $R_{\mathrm{m}}$ bins. Furthermore, we limit the analysis only to $\phi<200^{\circ}$, to exclude halo CMEs, being predominantly Earth- or anti-Earth- directed, i.e., to exclude events where projection effects are large.

\subsection{Simple $\phi$-bins}

In the first step we tried to sort the data into the $\Delta \phi=10^{\circ}$ bins, and for each bin we have drawn the $a(v)$ graphs independently to check if, and how, the slopes $\gamma_{1}$ depend on $\phi$. The procedure was applied to all velocity options and it turned out that the $\Delta \phi=10^{\circ}$ bins are generally too narrow, giving a clear $\gamma_{1}(\phi)$ relationship only in the case of the $\mathrm{n} 4 \mathrm{v} 3$-subset. The outcome is shown in Fig. 2a, where the $100^{\circ}<\phi<150^{\circ}$ and $150^{\circ}<\phi<$ $200^{\circ}$ data are merged, since for $\phi>100^{\circ}$ the $\Delta \phi=10^{\circ}$ bins are not abundant enough (see Fig. 10d in Appendix A) to provide statistically significant $a-v$ correlations.

Figure 2a shows that the parameter $\gamma_{1}$ is smaller for broader CMEs, exposing a distinct power-law-like dependence. On the other hand, as the bin-averaged values $\bar{\phi}$ increase, the binaveraged distances $\bar{R}_{\mathrm{m}}$ also increase because wider CMEs are usually traced to larger heights, i.e., $\phi$ and $R_{\mathrm{m}}$ are correlated
Table 3. Summary of power-law fit parameters $\left(k_{1}=\alpha_{\phi} \phi^{-\beta_{\phi}}, k_{1}=\right.$ $\left.\alpha_{R} R^{-\beta_{R}}\right)$ for $\Delta \phi=20^{\circ}$-bin sampling.

\begin{tabular}{cccc}
\hline \hline & $\mathrm{n} 4 \mathrm{v} 3$ & $\mathrm{n} 4 \mathrm{v} 5$ & $\mathrm{n} 4 \mathrm{vm}$ \\
\hline$\alpha_{\phi}$ & $0.22 \pm 0.03$ & $0.09 \pm 0.03$ & $0.17 \pm 0.03$ \\
$\beta_{\phi}$ & $0.50 \pm 0.04$ & $0.36 \pm 0.07$ & $0.69 \pm 0.09$ \\
$\alpha_{R}$ & $2.61 \pm 2.55$ & $0.52 \pm 0.57$ & $8.4 \pm 2.76$ \\
$\beta_{R}$ & $2.08 \pm 0.45$ & $1.49 \pm 0.49$ & $3.1 \pm 0.33$ \\
\hline
\end{tabular}

(see Fig. 11c in Appendix A). Consequently, one finds also a distinct $\gamma_{1}\left(R_{\mathrm{m}}\right)$ power-law-like dependence which is shown in Fig. 2b.

The decreasing trend of $\gamma_{1}(\phi)$ and $\gamma_{1}\left(R_{\mathrm{m}}\right)$ can be recognized also when other velocity options and data-subsets are utilized. However, statistically significant results $(P>95 \%$ confidence level) are achieved only if the bins are broadened to $\Delta \phi \geq 20^{\circ}$ bins. In the insets in Fig. 2 we show the outcome for $\mathrm{n} 4 \mathrm{v} 3$, n4v5, and $\mathrm{n} 4 \mathrm{vm}$ subsets, split into the $\Delta \phi=20^{\circ}$ bins. The power-law fit parameters are summarized in Table 3.

Table 3 and the insets in Fig. 2 illustrate that the fit parameters depend considerably on the velocity option - the errors of individual fit parameters are far smaller than the difference between different subsets. The power-law exponents $\beta_{R}$ received from the $\gamma_{1}(R)=\alpha_{R} R^{-\beta_{R}}$ fit vary from $\beta_{R}=1.5$ to 3.1 , whereas the exponents of the $\gamma_{1}(\phi)=\alpha_{\phi} \phi^{-\beta_{\phi}}$ fit vary from $\beta_{\phi}=0.36$ to 0.69 .

\subsection{Decoupling the $R_{m}-\phi$ crosstalk}

Due to the crosstalk of $\phi$ and $R_{\mathrm{m}}$, the presented simple-bin analysis does not really specify how $\gamma_{1}$ depends on $\phi$ at a given height, and vice versa, how $\gamma_{1}$ depends on $R_{\mathrm{m}}$ for CMEs of a given width class. So, in the next step we try to decouple the $\gamma(R, \phi)$ dependence. The straightforward procedure would be to select the data within a narrow interval of $R_{\mathrm{m}}$, and then to divide the data into a number of $\phi$-bins. Analogously, one could try to select CMEs having widths within a certain interval, and then to separate the data into $R_{\mathrm{m}}$-bins. However, in the former case the results turned out to be statistically significant only if the $R_{\mathrm{m}}$-interval includes at least $6<R_{\mathrm{m}}<12$, and analogously in the later case, if the width-interval embraces $30^{\circ}<\phi<90^{\circ}$. Thus, although in this way the $R_{\mathrm{m}}-\phi$ crosstalk is reduced to a certain degree, the effect of the $R_{\mathrm{m}}(\phi)$ dependence is still present because of too wide $\Delta R_{\mathrm{m}}$, or $\Delta \phi$ intervals used.

So we apply another, less stringent procedure, where we start by creating $\Delta \phi=20^{\circ}$ bins, and then proceed by eliminating in each bin CMEs with largest (smallest) values of $R_{\mathrm{m}}$, until $\bar{R}_{\mathrm{m}}$ in all $\phi$-bins attains a previously chosen value. This provides an insight into the $\gamma(\phi)$ dependence at a given height. Analogously, we group the data into $\Delta R_{\mathrm{m}}=2$ bins, and then successively eliminate wide (narrow) CMEs until all $R_{\mathrm{m}}$-bins show the same mean width $\bar{\phi}$. This provides an insight into the $\gamma(R)$ dependence for a chosen mean CME width.

Figure 3 illustrates how the $a-v$ relationship changes with increasing $\bar{R}_{\mathrm{m}}$. We show the n4v3-subset data in three 

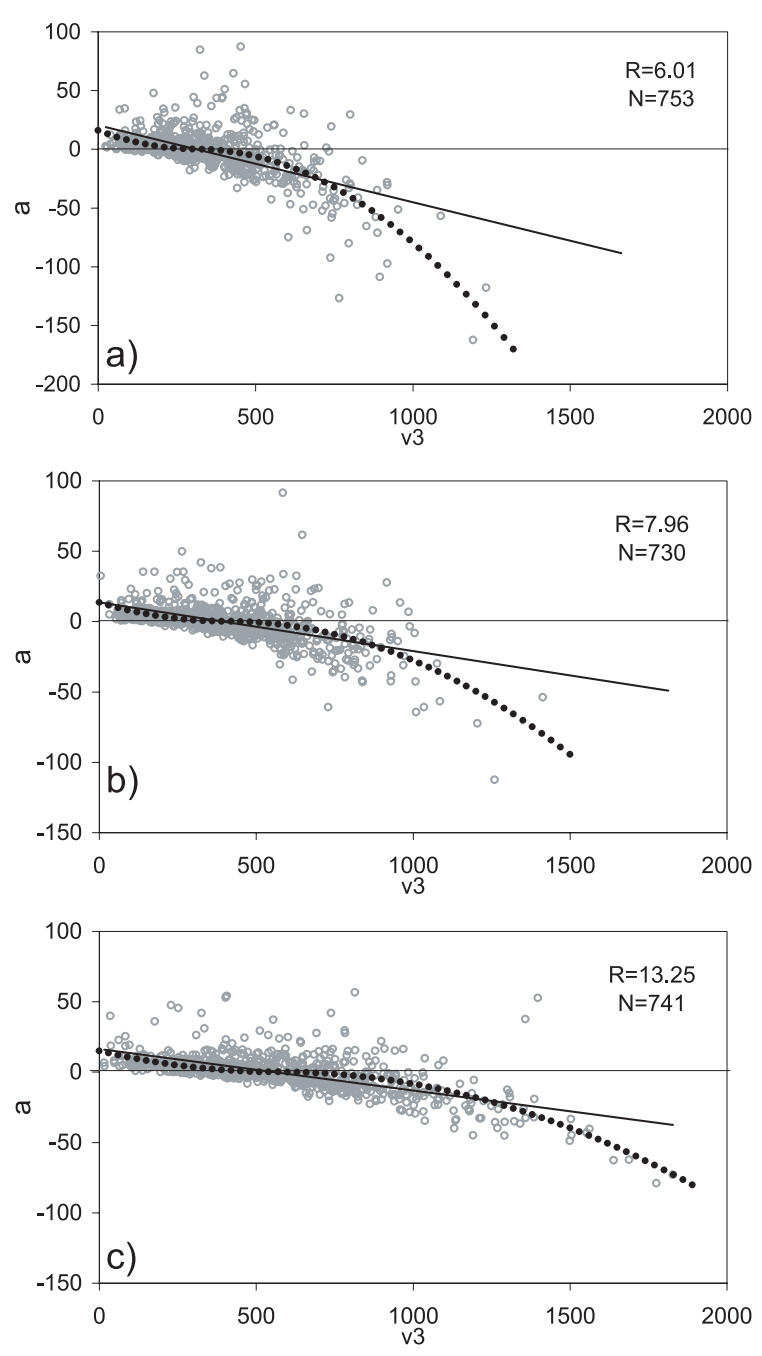

Fig. 3. The $a-v$ correlations shown for three $R_{\mathrm{m}}$-bins of the $\mathrm{n} 4 \mathrm{v} 3, \bar{\phi}=$ $60^{\circ}$, subset: a) $5<R_{\mathrm{m}}<7\left(\bar{R}_{\mathrm{m}}=6.01\right)$; b) $7<R_{\mathrm{m}}<9\left(\bar{R}_{\mathrm{m}}=\right.$ $7.96)$; c) $11<R_{\mathrm{m}}<15\left(\bar{R}_{\mathrm{m}}=13.25\right)$. The number of data points is indicated $(N)$.

different $\Delta R_{\mathrm{m}}=2$ bins, with the average width adjusted to the value $\bar{\phi}=60^{\circ}$ in each bin. The data are fitted by the linear and quadratic dependencies, and it turned out that the quadratic fits show a much larger statistical significance than in the case of complete data sets (Sect. 3) and the $R_{\mathrm{m}}-\phi$ coupled data (Sect. 4.1). Note that the linear, as well as the quadratic fits, become succesively less steep, whereas the $x$-axis intercepts $v_{0}$ shift to larger velocities. A similar behaviour can be recognized if one focuses on the lower boundary of the data (the "void" mentioned in Sect. 3)

In Fig. 4 the outcome for $n 4 v 3$-subset is summarized. The dependencies $\gamma_{1}(\phi)$ and $\gamma_{2}(\phi)$ are shown in Fig. 4a, where the $\phi$-bin averaged value of $R_{\mathrm{m}}$ is adjusted to the value $\bar{R}_{\mathrm{m}}=9$. The top panel depicts the linear-fit results, $\gamma_{1}(\phi)$, whereas in the bottom panel we present the quadratic-fit outcome, $\gamma_{2}(\phi)$. Similar results are obtained for $\bar{R}_{\mathrm{m}}=8$. For $\bar{R}_{\mathrm{m}}>9$ and $\bar{R}_{\mathrm{m}}<8$ the statistical significances of $a(v)$ fits in some $\phi$-bins become lower than $P<95 \%$ due to a small number of data, so the $\gamma(\phi)$ dependencies become unreliable.
In Fig. $4 \mathrm{~b}$ we show the $\gamma_{1,2}(R)$ dependence for $\bar{\phi}=30^{\circ}$ and $\bar{\phi}=60^{\circ}$. For $\bar{\phi}<30^{\circ}$ and $\bar{\phi}>60^{\circ}$ the results become unreliable due to the same reason as in the $\bar{R}_{\mathrm{m}}<8$ and $\bar{R}_{\mathrm{m}}>9$ case.

In Fig. $4 \mathrm{c}$ we show the $x$-axis intercept $v_{0}$ as a function of $\bar{R}_{\mathrm{m}}$, for $\bar{\phi}=30^{\circ}$ and $\bar{\phi}=60^{\circ}$, as found from the linearfitting (top) and the quadratic-fitting (bottom). The values of $v_{0}$ show a distinct increase with the radial distance. The obtained velocities are compared with the empirical solar wind model by Sheeley et al. (1997):

$w(R)=w_{\infty} \sqrt{1-\mathrm{e}^{-\left(R-R_{\mathrm{b}}\right) / R_{\mathrm{a}}}}$,

where $R_{\mathrm{b}}=2.8, R_{\mathrm{a}}=8.1$, and $w_{\infty}$ is the asymptotic value of the wind speed for $R \rightarrow \infty$, commonly taken to be $w_{\infty}=$ $400 \mathrm{~km} \mathrm{~s}^{-1}$. Obviously, the values of $v_{0}(R)$ are systematically larger than the solar wind speed $w(R)$, which will be discussed in Sect. 5.3.

The outcome for the $n 4 v m$-subset and the n4v5-subset is statistically less reliable than for the n4v3-subset. These options show a lower significance of $a-v$ correlations, and consequently larger errors of power-law fit coefficients for the $\gamma_{1,2}(\phi)$ and $\gamma_{1,2}(R)$ dependencies. Yet, the overall decreasing trend of $\gamma_{1}(\phi)$ and $\gamma_{1}(R)$ is well recognizable, although not as clear as in the $R_{\mathrm{m}}-\phi$ coupled case (insets in Fig. 2).

\section{Interpretation and discussion}

The acceleration-velocity relationship analysed in Sects. 3 and 4 indicates that the aerodynamic drag plays an important role in the dynamics of CMEs in the upper corona and the nearSun interplanetary space. In this section we interpret the obtained empirical relationships, relying on basic physical characteristics of the drag.

However, first we have to clarify some important issues considering the $a-v$ correlation as a statistical superposition of extracted kinematical parameters of different-width CMEs measured at different mean heights. Then we proceed by discussing the qualitative aspects of results that do not depend on the procedure and the velocity option. Eventually, we compare the results with some previous studies, and discuss the motion beyond $R=30$.

\subsection{Meaning of the $a-v$ relationship}

When the dynamics of an individual CME is considered, Eq. (1) relates the instantaneous acceleration with the instantaneous velocity, whereas actual CME measurements provide only the mean acceleration estimated over a wide distance range. Furthermore, CMEs have different widths $\phi$, and are measured at different $R_{\mathrm{m}}$, across different $\Delta R$. This means that the interaction of CMEs with the solar wind is (quantitatively) different for various "categories" of CMEs, as demonstrated in Sect. 4. For this reason it is instructive to pay attention to the meaning of the statistical outcome, taking into account the nature of individual measurements. Furthermore, one should bear in mind that CMEs move in various directions (different projection effects), the solar wind is highly inhomogeneous (different ambient densities and flow velocities for different CMEs), and the relative contribution of other forces might be different. 

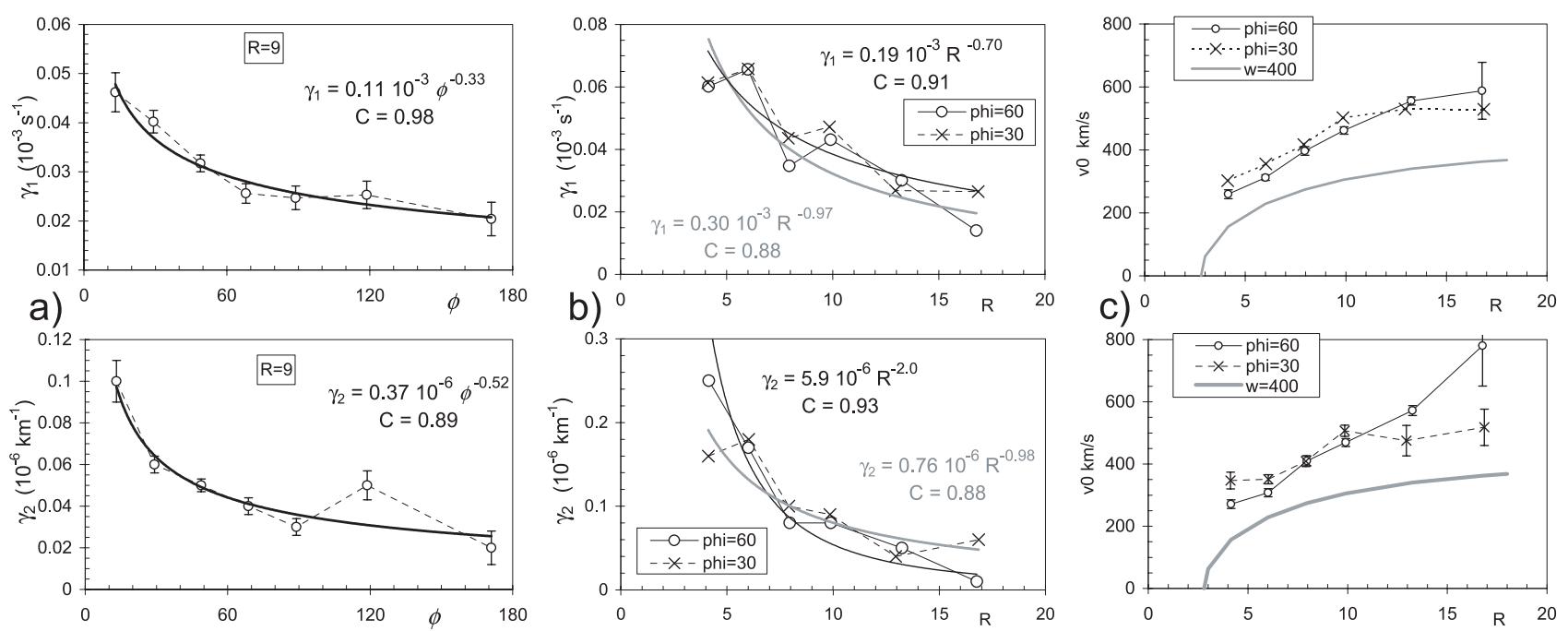

Fig. 4. Summary of the $a-v$ correlation parameters obtained for the $\mathrm{n} 4 \mathrm{v} 3$-subset. a) Slopes $\gamma_{1}$ (top) and $\gamma_{2}$ (bottom) shown as a function of bin-averaged widths $\bar{\phi}$ for $\bar{R}_{\mathrm{m}}=9$; b) slopes $\gamma_{1}$ (top) and $\gamma_{2}$ (bottom) shown as a function of bin-averaged distances $\bar{R}_{\mathrm{m}}$ for $\bar{\phi}=30^{\circ}$ (circles and fit shown in gray) and $\bar{\phi}=60^{\circ}$ (crosses and fit shown in black). c) The corresponding $x$-axis intercepts $v_{0}$ presented as a function of $R$ and compared with the empirical solar wind model by Sheeley et al. (1997) for $w_{\infty}=400 \mathrm{~km} \mathrm{~s}^{-1}$ (bold-gray line). In a) and b) the power-law least squares fits for $\gamma_{1,2}(\phi)$ and $\gamma_{1,2}(R)$ are shown together with the correlation coefficients $C$.
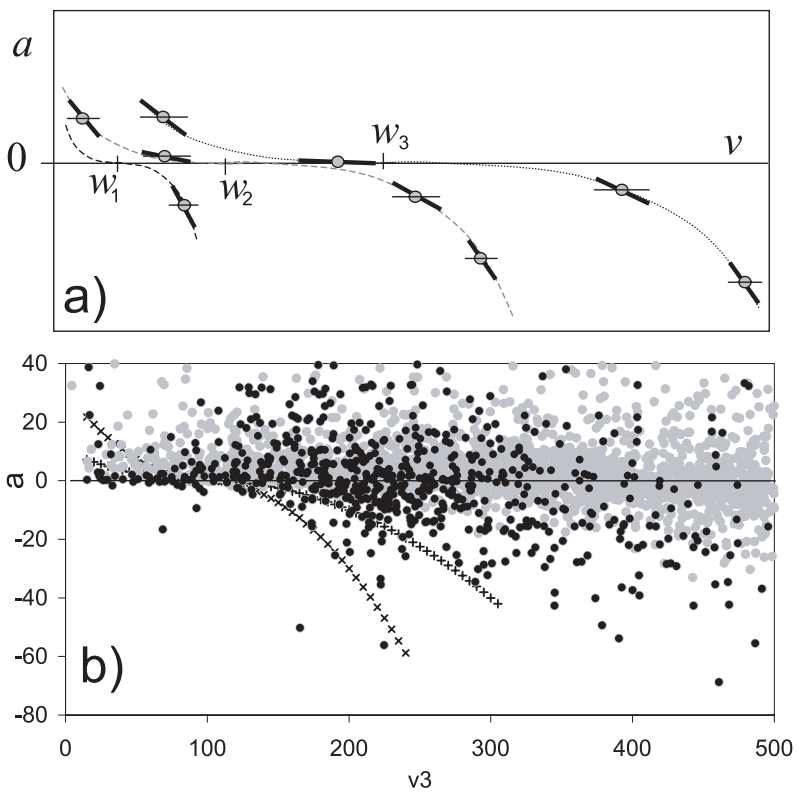

Fig. 5. a) A sketch showing three $a=-\gamma_{2}\left(v-v_{0}\right)|v-w|$ curves of different $\gamma_{2}$ and $w$, and the statistical outcome for a set of nine fictive-events that were traced during a limited time interval corresponding to bold segments on the $a(v)$-curves. The mean acceleration and velocity of a given event is marked by circle, and the velocity interval by thin horizontal line. b) Enlarged low-velocity part of the $a-v$ graph showing n4v3-subset data (gray), where the events with $R_{\mathrm{m}}<5$ are enhanced by black dots. Two quadratic $a(v)$-curves are drawn provisionally to depict the border of the "void".

In Fig. 5a we present schematically the physical background of the statistical relationship between the mean accelerations and velocities for an imaginary set of CMEs whose motion is dominated by the drag. We have drawn three $a=$ $a_{\mathrm{d}}=-\gamma_{2}(v-w)|v-w|$ curves, hereinafter abbreviated as $a(v)$-trajectories. The curves are characterized by a successively larger $w$ and smaller $\gamma_{2}$, representing the drag acceleration at different heights. Note that at a given ambient flow speed $w$, CMEs of different widths $\phi$ have different $\gamma_{2}$, which should be represented by a fan of curves crossing the $x$-axis at the given $w$. Furthermore, the presence of other forces shifts the curves up or down (see Sect. 5.3).

On the three depicted quadratic curves we have drawn the "real" $a(v)$-trajectories (bold segments of the curves) of several CMEs traveling at various velocities in different solar wind environments. CMEs that are faster than the solar wind $\left(v>w \Rightarrow a_{\mathrm{d}}<0\right)$ move in the left-up direction, whereas those with $v<w$, i.e., $a_{\mathrm{d}}>0$, move right-down. Note that the instantaneous acceleration of CMEs is changing. The measured mean acceleration and velocity are indicated by circles, whereas the change of the velocity is indicated by thin horizontal bars.

Figure 5a explains several aspects of the statistical results obtained in Sects. 3 and 4. First of all, it clarifies why the quadratic fits turned out to be worse than linear ones when the complete data set is considered. Measuring CMEs of different dimensions and at different heights smears out the basic $a(v)$ form into an inclined "cloud" of data points which is naturally better fitted by the straight line.

On the other hand, the analysed CMEs were measured only above the occulting disc of the LASCO-C2 coronagraph. So, the smearing effect is reduced below some $a(v)$-curve that is defined by the solar wind speed $w$ and $\gamma_{2}$ appropriate for $R_{\mathrm{m}}$ of CMEs measured at low heights across relatively short distance interval. Indeed, one finds a sharp-edged void at $a<$ $0, v<150 \mathrm{~km} \mathrm{~s}^{-1}$ (see Fig. 1) for any of the subsets and velocity options used. In Fig. 5b we show this part of the n4v3-subset $a-v$ graph enlarged, where two (provisionally chosen) quadratic $a(v)$-curves are inserted to depict roughly the limits within which lies the border of the void (pluses and 
crosses represent $\gamma_{2}=1$ and $3 \times 10^{-6} \mathrm{~km}^{-1}$, respectively; in both cases $w=100 \mathrm{~km} \mathrm{~s}^{-1}$ ). Going back to Fig. 3 one finds that as $R_{\mathrm{m}}$ increases, the edge of the void successively expands towards higher velocities and becomes less steep.

\subsection{Qualitative behaviour of the drag}

Let us now focus on the results that qualitatively do not depend on the procedure (the velocity option or sampling):

$i$ ) slopes of the $a-v$ correlations, $\gamma_{1}$ and $\gamma_{2}$, decrease with height and are smaller for wider CMEs;

ii) $x$-axis intercept of the $a-v$ correlation $\left(v_{0}=v_{a=0}\right)$ increases with height;

iii) $v_{0}(R)$ is systematically larger than the solar wind speed $w(R)$ for some $100-200 \mathrm{~km} \mathrm{~s}^{-1}$.

The parameter $\gamma_{2}$ in the drag acceleration, Eq. (1), depends on the CME cross-section $(A)$ and mass $(m)$, as well as on the ambient density $\left(\rho_{\mathrm{o}}\right)$ :

$\gamma_{2} \propto \frac{\rho_{\mathrm{o}} A}{m}$

(cf. Cargill et al. 1996). Employing a characteristic CME dimension, $d$, one can write for a given $R$ most generally:

$\gamma_{2} \propto \frac{\rho_{\mathrm{o}}}{\rho_{\mathrm{i}}} \frac{d^{2}}{d^{3}}=\frac{\rho_{\mathrm{o}}}{\rho_{\mathrm{i}} d}$,

where $\rho_{\mathrm{i}}$ stands for the internal CME density averaged over the CME body, and we substituted $m \propto \rho_{\mathrm{i}} d^{3}$ and $A \propto d^{2}$ (see Cargill 2004 for a more specific approach where the cylindrical shape is presumed). Assuming that $d$ is related to the angular width (e.g., $d \approx r \phi ; \phi \approx$ const.; Bothmer \& Schwenn 1994), one finds out that for a fixed distance $r, \gamma_{2}$ should be smaller for larger $\phi$. Indeed, the relationships exposed in Figs. $2 \mathrm{c}$ and $4 \mathrm{a}$ indicate such a dependence.

Here we have implicitly assumed that at a given height there is no statistical correlation between the CME density $\rho_{\mathrm{i}}$ and $d$. However, wider CMEs seem to be denser: they can be traced to larger distances (see Fig. 11c in Appendix A), implying that they are brighter, meaning also denser. This effect tends to steepen the slope of the $\gamma_{2}(\phi)$ dependence.

Equation (4) can be rewritten also as $\gamma_{2} \propto \rho_{\mathrm{o}} d^{2} / m$. Let us assume that $m(R) \approx$ const., i.e., that the mass loss from the prominence body and the mass pile-up at the leading edge of $\mathrm{CME}$, at least partly compensate each other. Furthermore, utilizing $d \propto r$, and taking into account that in the considered distance range the ambient density decreases with the height as, say, $\rho_{\mathrm{o}} \approx r^{-4}$ (see, e.g. Leblanc et al. 1998, or Vršnak et al. 2003) one finds roughly $\gamma_{2} \propto r^{-2}$. In other words, $\gamma_{2}$ has to decrease with the distance, consistent with Fig. 4b. At larger distances, where $\rho_{\mathrm{o}} \approx r^{-2}$, it should become approximately constant, except for the mass pile-up effect (for details see Cargill 2004).

\subsection{Influence of other forces}

The slopes of the empirical $\gamma_{1,2}(R)$ and $\gamma_{1,2}(\phi)$ dependencies obtained in Sects. 3 and 4 are quite sensitive on the sampling
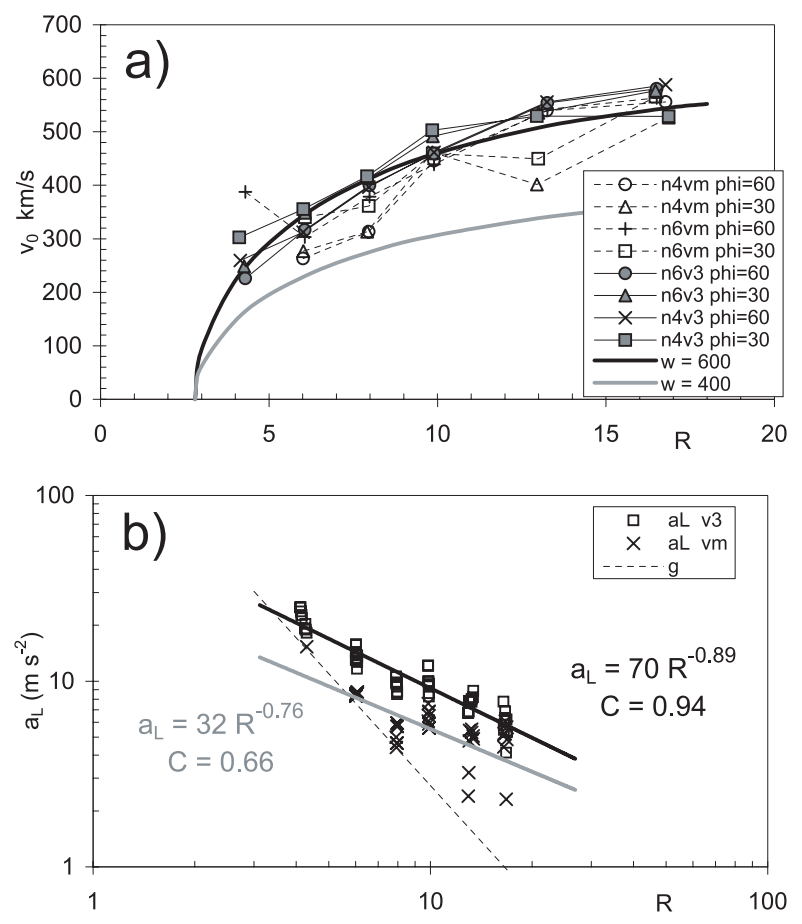

Fig. 6. a) The parameter $v_{0} \equiv v_{a=0}$ obtained from n4v3, n6v3, n4vm, and $n 6 \mathrm{vm}$ subsets presented as a function of $R$. The bin-averaged widths are adjusted to $\bar{\phi}=30^{\circ}$ and $\bar{\phi}=60^{\circ}$. The outcome is compared with the empirical solar wind model by Sheeley et al. (1997) for the asymptotic speed of $w_{\infty}=400$ and $600 \mathrm{~km} \mathrm{~s}^{-1}$ (bold-gray and bold-black curve, respectively). b) The inferred propelling force acceleration shown in the log-log graph for the $v_{3}$ (squares) and $v_{\mathrm{m}}$ (crosses) based subsets that are shown in a). The power-law fits are indicated in black and gray, respectively. The acceleration of gravity is indicated by thin-dashed line.

and velocity option. In such a situation a reliable quantitative comparison with a more detailed theoretical consideration would be exaggerated. On the other hand, the $x$-axis intercept $v_{0}$ in the $a-v$ correlation shows a much more coherent behaviour, so in the following we focus on this parameter.

In the absence of other forces the CME acceleration would be governed by Eq. (1), or in linear approximation by $a=$ $\gamma_{1}(v-w)$. Consequently, CMEs of a given $\bar{R}_{\mathrm{m}}$ would form the $a-v$ correlation that crosses the $x$-axis around $w\left(\bar{R}_{\mathrm{m}}\right)$. Here we find a distinct quantitative discrepancy: Fig. $4 \mathrm{c}$ shows that the $x$-axis intercepts $v_{0}(R)$ are systematically higher than the corresponding solar wind speeds $w(R)$.

To check this issue in more detail, in Fig. 6a we compare the $v_{0}(R)$ dependencies obtained from different data sets and velocity options, with the model values evaluated by Eq. (3). One finds out that velocities $v_{0}$ closely follow the solar wind model for the asymptotic speed of $w_{\infty} \approx 600 \mathrm{~km} \mathrm{~s}^{-1}$, which is considerably higher than the widely accepted value $w_{\infty}=400 \mathrm{~km} \mathrm{~s}^{-1}$. Going back to Table 1 and comparing the listed values with $w \approx 250 \mathrm{~km} \mathrm{~s}^{-1}$ evaluated from Eq. (3) for $\bar{R}_{\mathrm{m}} \approx 9$, one finds again that the values in Table 1 are larger for $\approx 150 \mathrm{~km} \mathrm{~s}^{-1}$. 


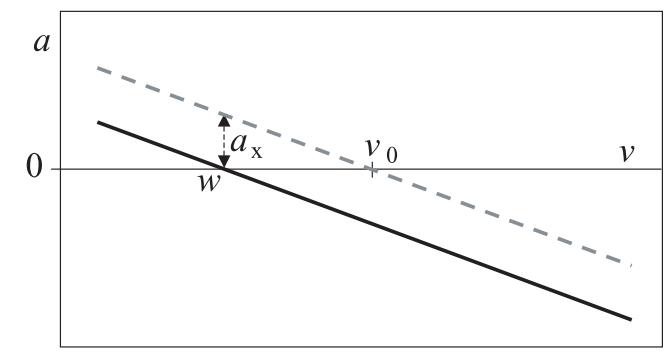

Fig. 7. Schematic presentation showing how the $a-v$ correlation (bold line) is shifted in the presence of a force which gives rise to the acceleration $a_{x}$. The resulting dependence (gray-dashed line) intercepts $x$-axis at $v_{0}>w$ if $a_{x}>0$, and at $v_{0}<w$ if $a_{x}<0$.

Such an excess can be interpreted in terms of a nonnegligible propelling force ${ }^{2}$. In Fig. 7 we schematically draw how the $a-v$ correlation is modified if the drag is not the only force acting on CMEs in the studied distance range. We consider only the simplest situation where the force (or several forces), giving rise to an acceleration $a_{x}$, does not statistically depend on $v$. Such a force does not affect the slope of the $a-v$ correlation - it only changes the $x$-axis intercept to $v_{0} \neq w$. If $a_{x}<0$ (like gravity), the intercept value decreases $\left(v_{0}<w\right)$, and if $a_{x}>0$ the intercept value increases to $v_{0}>w$. The acceleration $a_{x}$ can be determined from the difference $\Delta v=v_{0}-w$ as $a_{x}=\gamma_{1} \Delta v$ (Fig. 7).

In the following we consider the influence of gravity and the propelling (presumably Lorentz) force, giving rise to accelerations $g$ and $a_{\mathrm{L}}$. The total acceleration (force per unit mass) can be written as $a=a_{\mathrm{d}}-g+a_{\mathrm{L}}$, where $a_{\mathrm{d}}$ represents the drag acceleration. So, the additional net acceleration $a_{x}=a_{\mathrm{L}}-g$ can be estimated as $a_{\mathrm{L}}-g=\gamma_{1} \Delta v$. Since the values $\gamma_{1}(R)$ are known from observations (e.g., Fig. 4b) and $\Delta v(R)$ can be estimated using some solar wind model (e.g., Eq. (3)), the Lorentz force acceleration can be evaluated as $a_{\mathrm{L}}=\gamma_{1} \Delta v+g$, where $g(R)=274 / R^{2}$.

Note that by using $g(R)=274 / R^{2}$ we neglect the buoyancy - assuming $\rho_{\mathrm{o}} \approx \rho_{\mathrm{i}}$ one gets for the net acceleration of gravity $a_{\mathrm{g}}=0$. The value of $a_{\mathrm{L}}$ inferred by using $g$ can be considered as an upper limit. Another drawback of the procedure lies in the fact that faster CMEs have on average larger $R_{\mathrm{m}}$. So, the slope of the $a-v$ correlation would be altered since $g(R)$ decreases: the influence of $g$ is, statistically, smaller at larger velocities and the $a-v$ correlation becomes less steep ( $\gamma_{1}$ decreases). Analogously if $a_{\mathrm{L}}(R)$ decreases, the $a-v$ correlation becomes steeper. In principle, it would be possible to perform an iterative procedure to estimate more precisely $a_{\mathrm{L}}(R)$, but bearing in mind the accuracy of the observational input and other drawbacks of the applied procedure (see Appendix B), such a treatment would be exaggerated.

For the matter of illustration, let us first take $\gamma_{1} \approx 0.02 \times$ $10^{-3} \mathrm{~s}^{-1}$ and $v_{0}=350-400 \mathrm{~km} \mathrm{~s}^{-1}$ ("average" values from

\footnotetext{
${ }^{2}$ Ruždjak et al. (2004) offered also an alternative explanation, where the CME-wind coupling is governed by the fast solar wind. Yet, most of CMEs are launched in the streamer belt, and their mean width is relatively small (see Appendix A), so such an ad hoc interpretation in fact does not provide a satisfying explanation.
}

Table 1). Using $w \approx 250 \mathrm{~km} \mathrm{~s}^{-1}$ at $\bar{R}_{\mathrm{m}}=9$ as obtained from Eq. (3), one finds $\Delta v=v_{0}-w=100-150 \mathrm{~km} \mathrm{~s}^{-1}$. Utilizing these values one receives $a_{\mathrm{L}}-g=\gamma_{1} \Delta v=2-3 \mathrm{~m} \mathrm{~s}^{-2}$, i.e., $a_{\mathrm{L}} \approx 6 \mathrm{~m} \mathrm{~s}^{-2}$, since $g_{R=9}=3.4 \mathrm{~m} \mathrm{~s}^{-2}$.

Let us now evaluate $a_{\mathrm{L}}\left(\bar{R}_{\mathrm{m}}\right)=\gamma_{1} \Delta v+g$ using the differences $\Delta v\left(\bar{R}_{\mathrm{m}}\right)=v_{0}-w\left(\bar{R}_{\mathrm{m}}\right)$ and slopes $\gamma_{1}\left(\bar{R}_{\mathrm{m}}\right)$ for all individual $R$-bins considered in Sects. 3. and 4.

The outcome is shown in Fig. $6 \mathrm{~b}$ for all subsets that are utilized in Fig. 6a. Bearing in mind all drawbacks of the procedure, one can say that the propelling-force acceleration, i.e., the propelling force per unit mass, at $R \approx 10$ amounts to $a_{\mathrm{L}} \approx$ $5-10 \mathrm{~m} \mathrm{~s}^{-2} \equiv 5-10 \mathrm{~N} \mathrm{~kg}^{-1}$. The data show a decreasing trend which can be roughly expressed as $\approx R^{-1}$. The values based on the velocity $v_{3}$ are larger than those obtained by using $v_{\mathrm{m}}$. This is primarily a result of the steeper slope $\gamma_{1}$ and somewhat larger $v_{0}$ of the $a-v_{3}$ correlation (Sect. 5.1).

Let us note that using the isothermal $\left(T=10^{6} \mathrm{~K}\right)$ solar wind model by Parker (1958; see also Mann et al. 2003) instead of Eq. (3), one gets a 30-50\% larger $a_{x}$ (resulting in 10-30\% larger $a_{\mathrm{L}}$ ), but the slope of $a_{x}(R)$ remains approximately $R^{-1}$.

We emphasize the statistical nature of Fig. 6b, i.e., it does not represent an average behaviour of the propelling force $a_{\mathrm{L}}(R)$ in a "typical" CME. It rather shows that the propelling force is on average weaker at larger distances, being consistent with the anticipated behaviour of the Lorentz force (Sect. 1).

There is also another way to estimate the quantity $a_{\mathrm{L}}=$ $a+g-a_{\mathrm{d}}$. The drag acceleration for a given CME can be evaluated from $a_{\mathrm{d}} \approx-\gamma_{1}(v-w)$ by substituting the velocity $v=v_{\mathrm{m}}$, $v_{3}$, or $v_{5}$, and the model-value $w=w\left(R_{\mathrm{m}}\right)$. For estimating $\gamma_{1}$ one can take the empirical scaling law $\gamma_{1}=1.16 R^{-1.35} \times 10^{-3} \mathrm{~s}^{-1}$ found by a different procedure by Vršnak (2001a). Finally, using the measured values $a_{\mathrm{m}}$ and $g\left(R_{\mathrm{m}}\right)=274 / R_{\mathrm{m}}^{2}$ one can estimate $a_{\mathrm{L}}$ for each event individually.

The calculated values $a_{\mathrm{d}}$ are shown in Fig. 8a as a function of $v_{3}$ (a similar outcome is also found using $v_{\mathrm{m}}$ or $v_{5}$ ). The overall anticorrelation pattern in Fig. 8a is similar to that exposed by Fig. 1, justifying the proposed interpretation in terms of the drag force, and on the other hand confirming reliability of the empirical scaling law $\gamma(R)$ obtained by Vršnak (2001a). The slopes of the linear fits in Figs. $8 \mathrm{a}$ and 1 are similar, whereas there is a difference in the $x$-axis intercept $v_{0}$. The linear fit in Fig. 8a crosses the $x$-axis at $v_{0} \approx 250 \mathrm{~km} \mathrm{~s}^{-1}$, i.e., it is lower for $\Delta v_{0} \approx 150 \mathrm{~km} \mathrm{~s}^{-1}$. Again, this can be attributed to the existence of $a_{x}>0$. Taking for the slope of the $a_{\mathrm{d}}(v)$ correlation $\gamma_{1}=-0.026 \times 10^{-3} \mathrm{~s}^{-1}$ (see the fit in Fig. 8a) one finds $a_{x}=\gamma_{1} \Delta v_{0}=+3.9 \mathrm{~m} \mathrm{~s}^{-2}$, and assuming $a_{x}=a_{\mathrm{L}}-g$ one receives $a_{\mathrm{L}}\left(R_{\mathrm{m}}\right)=7.3 \mathrm{~m} \mathrm{~s}^{-2}$, consistent with the previous estimates.

Another difference found in Figs. 1 and 8a is a considerably larger scatter of the data in Fig. 1. Firstly, this can be attributed to a variety of the propelling force strengths. Furthermore, the differences in CME widths are not included in the evaluation of $a_{\mathrm{d}}$ since the used scaling law by Vršnak (2001a) does not provide that. Finally, note that in the low-velocity regime, $v<$ $150 \mathrm{~km} \mathrm{~s}^{-1}$, the accelerations appear in Fig. 8a down to $a_{\mathrm{d}} \approx$ $-10 \mathrm{~m} \mathrm{~s}^{-2}$, whereas in Fig. 1 (see also Fig. 5) the upper edge 
of the void is at $a=0$. Such a difference is also consistent with the previous estimates of the propelling force.

In Fig. 8b we compare the distributions $N\left(a_{\mathrm{d}}\right)$ and $N\left(a_{\mathrm{L}}\right)$ for the n6v3-subset (see also the the distribution of measured accelerations, $N\left(a_{\mathrm{m}}\right)$ shown in Fig. 10f in Appendix A). In addition, we present in the inset the distribution $N(g)$, where $g=$ $g\left(R_{\mathrm{m}}\right)$. Mean values are: $\bar{a}_{\mathrm{d}}=-5.4 \pm 9.7 \mathrm{~m} \mathrm{~s}^{-2}, \bar{a}_{\mathrm{L}}=10.0 \pm$ $13.9 \mathrm{~m} \mathrm{~s}^{-2}$, and $\bar{g}=-5.2 \pm 4.6 \mathrm{~m} \mathrm{~s}^{-2}$, which explains why CMEs in the upper corona and near-Sun interplanetary space show on average $\bar{a}_{\mathrm{m}} \approx 0$ (Appendix A). We emphasize that very similar distributions are obtained utilizing any other subset or velocity option. The drag acceleration is dominantly negative, i.e., most events are faster than the solar wind. On the other hand, in the majority of events, $a_{\mathrm{L}}$ lies between 0 and $20 \mathrm{~m} \mathrm{~s}^{-2}$, consistent with Fig. $6 \mathrm{~b}$. The distribution indicates that there is a considerable fraction (18\%) of events where $a_{\mathrm{L}}$ appears to be larger than $20 \mathrm{~m} \mathrm{~s}^{-2}$ (see also Vršnak 2001b). In extreme cases $(2 \%) a_{\mathrm{L}}$ is larger than $50 \mathrm{~m} \mathrm{~s}^{-2}$ (to be studied in detail in a separate paper). In $17 \%$ of events the inferred values of $a_{\mathrm{L}}$ are negative, with $5 \%$ showing $a_{\mathrm{L}}<-10 \mathrm{~m} \mathrm{~s}^{-2}$. Note that the $a_{\mathrm{L}}<0$ regime is revealed by the "fall-back" events described by Wang \& Sheeley (2002) and by the existence of an upper equilibrium observed in some events (Vršnak et al. 1990; Vršnak 2001b). Such a behaviour is anticipated in the flux rope model by Vršnak (1990).

Finally, let us emphasize one potentially important sideresult of the presented analysis. None of the presented graphs (including those in Appendix A) shows any kind of grouping of CMEs that would confirm the existence of two (or more) distinct classes of events regarding the velocity and/or acceleration behaviour, as proposed by some authors who studied small samples of preferentially large CMEs (see, e.g., MacQueen \& Fisher 1983, or Andrews \& Howard 2001). Rather, the distribution of CMEs in the $a-v$ space indicates that there is a continuum of events over the actual acceleration and velocity range.

\subsection{Comparison with previous studies}

Values of the exponent $\beta_{R}$ in the $\gamma_{1} \propto R^{-\beta_{R}}$ scaling received in Sect. 4.1 , can be directly compared with the result by Vršnak (2001a) since the $R_{\mathrm{m}}-\phi$ crosstalk was implicitly present therein too. The obtained values $\beta_{R}=1.5-3.1$ are larger than $\beta_{R}=1.35$ found by Vršnak (2001a), indicating a considerably steeper decrease of $\gamma_{1}$. However, it should be noted that the results by Vršnak (2001a) were based on a limited set of only 12 events, all of which showed an exponential-like decay of velocity, indicating that the drag is a dominant force. Furthermore, seven of these events were measured at low heights by tracing $\mathrm{H} \alpha$ features, i.e., the deceleration rates could be higher for leading edges of CMEs.

In Fig. 9 we compare the slopes of the $a-v$ correlations obtained herein with the scaling law $\gamma_{1}(R)$ established by Vršnak (2001a), and with the results for the interplanetary space obtained by Gopalswamy et al. (2000) and Gopalswamy et al. (2001b). The values for the complete n4v3-subset and n6v3-subset are positioned at $R=\bar{R}_{\mathrm{m}}=9$ and overlap each other. The slopes from Gopalswamy et al. (2000) and
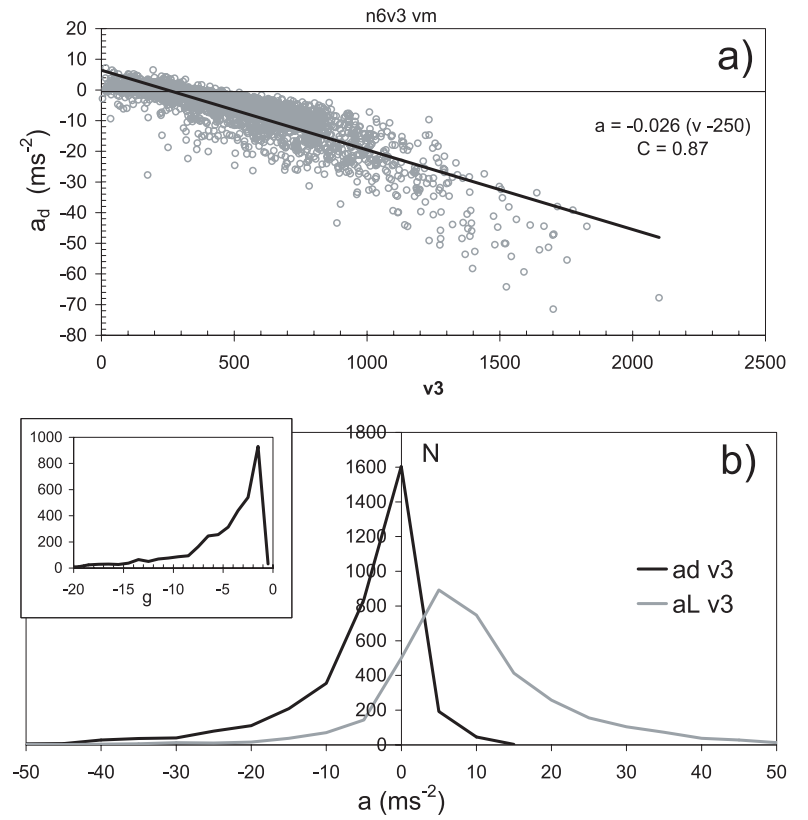

Fig. 8. a) Drag accelerations estimated using $v_{\mathrm{m}}$ for n6v3-subset, shown in the $a_{d}-v_{3}$ space. The corresponding linear least squares fit is indicated. b) The n6v3-subset distribution of estimated drag accelerations (black) and the corresponding inferred propelling force accelerations $a_{\mathrm{L}}$ (gray). The bin width is $5 \mathrm{~m} \mathrm{~s}^{-2} .23(0.6 \%)$ events in $a_{\mathrm{d}}$ distribution and $47(1.3 \%)$ in $a_{\mathrm{L}}$ distribution are out of the scale. In the inset the distribution of the acceleration of gravity $g$ is shown for bins of $1 \mathrm{~m} \mathrm{~s}^{-2}$.

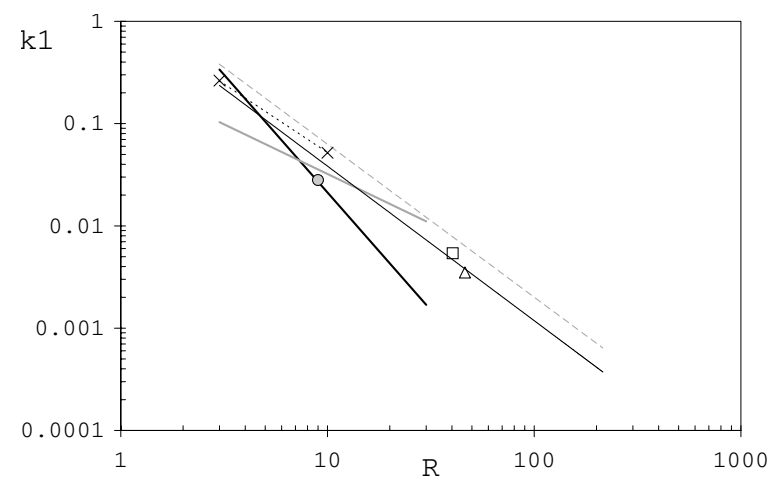

Fig. 9. The slopes $k_{1}=10^{3} \gamma_{1}$ of the linear $a-v$ correlations, presented as a function of radial distance: circle - n6v3-subset; triangle - Goplaswamy et al. (2001b); square - Gopalswamy et al. (2000); crosses connected by the dotted line - Vršnak (2001a). The powerlaw fit to these data-points reads $k_{1}=1.2 R^{-1.5}$ and is drawn by the thin-black line. The gray dashed line shows the scaling $k_{1}=2 R^{-1.5}$ inferred by Vršnak \& Gopalswamy (2002). The bold-black and boldgray lines show power-law fits from Fig. 2b and from Fig. 4b-top for $\bar{\phi}=60^{\circ}$, respectively.

Gopalswamy et al. (2001b) are drawn at $R=40$ and $R=46$, respectively, corresponding to the geometrical mean (see the argumentation in Vršnak 2001a) of the considered distance ranges ( 0.76 and $1 \mathrm{au}$, respectively). The fit through the presented data-points matches quite closely to the scaling $k_{1}=$ $2 R^{-1.5}$ that was inferred by Vršnak \& Gopalswamy (2002) from the Sun-Earth transit times. 
Table 4. Statistics of CMEs faster than solar wind at $R \geq 25$.

\begin{tabular}{lcccc}
\hline \hline$v^{*}\left(\mathrm{~km} \mathrm{~s}^{-1}\right)$ & $N$ & $\%$ & $\bar{\phi}$ & $\bar{a}_{\mathrm{m}}$ \\
\hline 500 & $374(215)$ & $8(57)$ & 137 & +0.9 \\
600 & $299(177)$ & $7(59)$ & 143 & +1.4 \\
800 & $175(107)$ & $4(61)$ & 166 & +3.1 \\
1000 & $88(53)$ & $2(59)$ & 196 & +5.2 \\
\hline
\end{tabular}

\subsection{Motion of CMEs beyond $R=30$}

Figure $8 \mathrm{~b}$ indicates that in the $R=2-30$ range the majority of events has already more or less adjusted to the solar wind speed since the $N\left(a_{\mathrm{d}}\right)$ distribution (Fig. 8) is sharply peaked at $a_{\mathrm{d}}=0$. A similar conclusion can be drawn from Fig. 1a, since most events are grouped around $a=0, v \approx 400 \mathrm{~km} \mathrm{~s}^{-1}$.

Let us now inspect the distribution of speeds $v_{\mathrm{e}}$ estimated at the time of last measurement (see Sect. 2) for the events that were traced to $R_{\mathrm{e}} \geq 25$. We find that only 374 out of $4463 \mathrm{n} 4 \mathrm{v} 3$-events $(8 \%)$ had $v_{\mathrm{e}}>500 \mathrm{~km} \mathrm{~s}^{-1}$. The number of events with velocities above some referent value, $v_{\mathrm{e}}>v^{*}$, decreases as we increase the limit $v^{*}$. The events with $v_{\mathrm{e}}>$ $v^{*}$ are on average much broader than the rest of the sample. Furthermore, they show a positive mean acceleration, being statistically larger for faster (and broader) CMEs. The $\mathrm{n} 4 \mathrm{v} 3$-subset statistics is summarized in Table 4 for different values of $v^{*}$. The number $(N)$ and the percentage $(\%)$ of the $v>v^{*}$ events is presented in the 2 nd and 3rd column. The values in brackets provide the number (and relative percentage) of $a_{\mathrm{m}}>0$ events. The average values of widths and accelerations are given in the last two columns. Note that more than half of the $v_{\mathrm{e}}>v^{*}$ events are characterized by $a_{\mathrm{m}}>0$. In some cases it is larger than $a_{\mathrm{m}}=50 \mathrm{~m} \mathrm{~s}^{-2}$.

Such a behaviour has important implications for the prediction of the $1 \mathrm{au}$ transit. In most CMEs the propelling force ceases, and the velocity becomes adjusted to the solar wind speed, already below $R=30$. The arrival of these events to the Earth (if they preserve their integrity) can be estimated to a sufficient accuracy utilizing the $v=$ const. $=400 \mathrm{~km} \mathrm{~s}^{-1}$ approximation. However, for very fast/wide CMEs, which in fact are the most geo-effective events (Zhang et al. 2003), the prediction based on LASCO observations is more difficult since the action of the propelling force is prolonged in such events. To be successful in predicting the 1 au transit, a higher degree polynomial fit to the $R(t)$ LASCO-data should be tried, to estimate if possible, how the acceleration changes. This might give a clue whether the event had entered the drag-dominated regime or not. If yes, the interplanetary motion of the CME should be calculated using the velocity at $R=30$ as the initial velocity and utilizing the scaling $\gamma_{\phi}(R)$ for the corresponding $\phi$-bin. Then, the equation of motion given by Eq. (1) should be solved numerically, in the manner demonstrated by Vršnak \& Gopalswamy (2002). Note that events of this kind should have the smallest deceleration rates since they are broad, which implies a relatively small $\gamma_{2}$.

The prolonged action of the propelling force in fast/wide CMEs is consistent with the observations based on the interplanetary scintillation (IPS). For example, Manoharan et al. (2001) have analysed the IP propagation of the CME associated with the large flare of 14 July 2000 . Until $R \approx 100$ the speed of ejection was almost constant $\left(v \propto R^{-0.08}\right)$, and only beyond this distance it started decelerating more rapidly, as $v \propto R^{-0.72}$. In such cases, the Thompson scattering measurements by the Solar Mass Ejection Imager (SMEI) combined with IPS observations (Jackson et al. 2003; Eyles et al. 2003) could be essential for the understanding of the IP propagation of CMEs and a successful prediction of 1 au transit time.

\section{Conclusion}

We have shown that there is a well defined anticorrelation between the acceleration and velocity of CMEs measured in the range $2<R<30$. The relationship can be comprehended as the statistical outcome for a sample of CMEs whose motion is significantly influenced by the aerodynamic drag: Only $14 \%$ of fast $\left(v_{3}>1000 \mathrm{~km} \mathrm{~s}^{-1}\right)$ CMEs are still speeding up, and $7 \%$ of slow CMEs $\left(v_{3}<150 \mathrm{~km} \mathrm{~s}^{-1}\right)$ show (only a weak) deceleration.

However, the existence of fast events whose velocity is still increasing suggests that in a certain fraction of events the propelling force is not negligible. On the other hand, the deceleration of slow events, and existence of the $a_{\mathrm{L}}<0$ events in the $N\left(a_{\mathrm{L}}\right)$ distribution (Fig. 8b) indicates that the deceleration in these events could be caused not only by the gravity, but also by the Lorentz force (see also Wang \& Sheeley 2002), which under special conditions might change its sign after passing an upper equilibrium point (Vršnak 1990).

The overall behaviour of the parameter $\gamma_{2}$, decreasing with the distance and being of the order of $0.1 \times 10^{-6} \mathrm{~km}^{-1}$ at $R \approx 10$, is very similar to that obtained by Vršnak (2001a). In addition, we found herein that the value of $\gamma_{2}$ is smaller for broader CMEs when measured at the same $R_{\mathrm{m}}$. On the other hand, the slope of the linear $a-v$ correlation at $R_{\mathrm{m}} \approx 10$ ranges between $\gamma_{1}=0.01-0.03 \times 10^{-3} \mathrm{~s}^{-1}$, which is smaller by roughly a factor of two than found by Vršnak (2001a).

The results indicate that in the majority of events the $v \approx w$ regime is achieved already in the LASCO field-of-view, i.e., below $R<30$. Only CMEs with prolonged action of the propelling force come into the $v \approx w$ regime beyond $R>30$, but the events with, e.g., $v>500 \mathrm{~km} \mathrm{~s}^{-1}$ at $R>25$ represent less than $10 \%$ of the CME population.

Acknowledgements. We are grateful to the LASCO-SOHO team for operating the instruments and performing the basic data reduction, and especially, for the open data policy.

\section{References}

Andrews, M. D., \& Howard, R. A. 2001, Space Sci. Rev., 95, 147

Anzer, U. 1978, Sol. Phys., 57, 111

Bothmer, V., \& Schwenn, R. 1994, Space Sci. Rev., 70, 215

Brueckner, G. E., Howard, R. A., Koomen, M. J., et al. 1995, Sol. Phys., 162, 357

Cargill, P. J. 2004, Sol. Phys., submitted

Cargill, P. J., \& Schmidt, J. M. 2002, Ann. Geophys., 20, 879

Cargill, P. J., Chen, J., Spicer, D. S., \& Zalesak, S. T. 1996, Geophys. Res., 101, 4855 
Chen, J. 1996, J. Geophys. Res., 101, 27499

Chen, J., \& Krall, J. 2003, J. Geophys. Res., 108, A11, 1410, 10.1029/2003JA009849

Eyles, C. J., Simnett, G. M., Cooke, M. P., et al. 2003, Sol. Phys., 217, 319

Forbes, T. G. 2000, J. Geophys. Res., 105, 23153

Gopalswamy, N. 2002, in Solar-terrestrial magnetic activity and space environment, COSPAR Colloquia Series, Vol. 14, ed. H. N. Wang, \& R. L. Xu, 157

Gopalswamy, N., Lara, A., Lepping, R. P., et al. 2000, Geophys. Res. Lett., 27, 145

Gopalswamy, N., Yashiro, S., Kaiser, M. L., Howard, R. A., \& Bougeret, J.-L. 2001a, J. Geophys. Res., 106, 29219

Gopalswamy, N., Lara, A., Yashiro, S., Kaiser, M. L., \& Howard, R. A. 2001b, J. Geophys. Res., 106, 29207

Gopalswamy, N., Shimojo, M., Lu, W., et al. 2003, ApJ, 586, 562

Gosling, J. T. 1990, in Physics of magnetic flux ropes, Geophys. Monogr. Ser., ed. C. T. Russell, E. R. Priest, \& L. C. Lrr, AGU, Washington, DC, 58, 343

Harrison, R. A. 1995, ApJ, 304, 585

Hundhausen, A. J. 1993, J. Geophys. Res., 98, 13177

Hundhausen, A. J., Burkepila, J. T., \& St. Cyr, O. C. 1994, J. Geophys. Res., 99, 6543

Jackson, W. D. 1975, Classical Electrodynamics (New York: Wiley \& Sons)

Jackson, B. V., Hick, P. P., \& Buffington, A. 2003, in Proc. ISCS 2003 Symp. Solar Variability as an input to the Earth's Environment, ed. A. Wilson, ESA SP-535, 823

Landau, L. D., Lifshitz, E. M., \& Pitaevski, L. P. 1984, Electrodynamics of Continuous Media, 2nd edition (Oxford: Pergamon Press)

Leblanc, Y., Dulk, G. A., \& Bougeret, J.-L. 1998, Sol. Phys., 183, 165

Lin, J., \& Forbes, T. G. 2000, J. Geophys. Res., 105, 2375

MacQueen, R. M., \& Fisher, R. R. 1983, Sol. Phys., 89, 89

Mann, G., Klassen, A., Aurass, H., \& Classen, H.-T. 2003, A\&A, 400, 329
Manoharan, P. K., Tokumaru, M., Pick, M., et al. 2001, ApJ, 559, 1180

Martens, P. C. H. 1987, Sol. Phys., 107, 95

Moon, Y.-J., Choe, G. S., Wang, H., et al. 2002, ApJ, 581, 694

Parker, E. N. 1958, ApJ, 128, 664

Ruždjak, D., Vršnak, B., \& Sudar, D. 2004, Solar magnetic phenomena, ed. A. Hanslmeier, A. Veronig, \& M. Messerotti (Kluwer), in press

Sheeley, N. R., Jr., Wang, Y.-M., Hawley, S. H., et al. 1997, ApJ, 484, 472

St. Cyr, O. C., Burkepile, J. T., Hundhausen, A. J., \& Lecinski, A. R. 1999, J. Geophys. Res., 104, 12493

St. Cyr, O. C., Howard, R. A., Sheeley, N. R., et al. 2000, J. Geophys. Res., 105, 18169

Vandas, M., Fisher, S., Dryer, M., Smith, Z., \& Detman, T. 1995, J. Geophys. Res., 100, 12285

Vandas, M., Fisher, S., Dryer, M., Smith, Z., \& Detman, T. 1996, J. Geophys. Res., 101, 2505

Vršnak, B. 1990, Sol. Phys., 129, 295

Vršnak, B. 2001a, Sol. Phys., 202, 173

Vršnak, B. 2001b, J. Geophys. Res., 106, 25249

Vršnak, B., \& Gopalswamy, N. 2002, J. Geophys. Res., 107, 10.1029/2001AJ000120

Vršnak, B., Ruždjak, V., Brajša, R., \& Zloch, F. 1990, Sol. Phys., 127, 119

Vršnak, B., Magdalenić, J., \& Zlobec, P. 2003, A\&A, 413, 753

Vršnak, B., Ruždjak, D., Sudar, D., \& Gopalswamy, N. 2003, in Proc. ISCS 2003 Symp. Solar Variability as an input to the Earth's Environment, ed. A. Wilson, ESA SP-535, 517

Wang, Y.-M., \& Sheeley, N. R., Jr. 2002, ApJ, 567, 1211

Wang, H., Qiu, J., Ju, J., \& Zhang, H. 2003, ApJ, 593, 564

Webb, D. F., Forbes, T. G., Aurass, H., et al. 1994, Sol. Phys., 153, 73

Zhang, J., Dere, K. P., Howard, R. A., \& Bothmer, V. 2003, ApJ, 582, 520 


\section{Online Material}




\section{Appendix A: Properties of the sample}

The events which were measured at $n \geq 4$ instants, show the distribution centered at $a_{\mathrm{m}} \approx 0$, with the standard deviation $\sigma_{\mathrm{a}}=31 \mathrm{~m} \mathrm{~s}^{-2}$. The average initial, mean, and final distances amount to $\bar{R}_{\mathrm{b}}=3.4 \pm 1.0, \bar{R}_{\mathrm{m}}=9 \pm 4$, and $\bar{R}_{\mathrm{e}}=14 \pm 7$, respectively.

In Fig. 10 we present the distribution of initial radial distances $R_{\mathrm{b}}$, the distance range $\Delta R=R_{\mathrm{e}}-R_{\mathrm{b}}$, mean radial distances $R_{\mathrm{m}}$, widths $\phi$, mean velocities $v_{\mathrm{m}}$, and mean accelerations $a_{\mathrm{m}}$, for the $\mathrm{n} 4 \mathrm{v} 3$-subset. Note that most often the events start at $3 \leq R<4$. The n4v3-subset contains $280 \mathrm{CME}$ events $(6 \%)$ of the angular width larger than $180^{\circ}$, out of which 184 were classified as $360^{\circ}$-halo CMEs (4\%). The basic statistical parameters of the distributions are given in Table 5.

The average error of the accelerations obtained from the 2nd degree polynomial fits is $\bar{M}_{\mathrm{a}}=7.6 \mathrm{~m} \mathrm{~s}^{-2}$ in the n4-set, and it drops to $4.7 \mathrm{~m} \mathrm{~s}^{-2}$ in the n6-set. The fraction of events with errors $\bar{M}_{\mathrm{a}}>10 \mathrm{~m} \mathrm{~s}^{-2}$ is $18 \%$ and $10 \%$ for the two data-sets, respectively.

The parameters $R_{\mathrm{m}}, \phi$, and $v_{\mathrm{m}}$ are mutually correlated (see also, e.g., Hundhausen et al. 1994), which is shown in Fig. 11, where the data are presented together with the linear least squares fits and corresponding correlation coefficients $(C)$. We emphasize that the $\phi\left(R_{\mathrm{m}}\right)$ correlation does not represent the broadening of CMEs with the height, but rather demonstrates that larger CMEs can be traced to larger distances, i.e., that broader CMEs are generally brighter. Similar holds also for the $v_{\mathrm{m}}\left(R_{\mathrm{m}}\right)$ relationship - faster CMEs are on average brighter and can be followed to larger heights. No statistically significant correlation between $a_{\mathrm{m}}$ and $R_{\mathrm{m}}$, or $\phi$ is found $(C<0.01)$.

The basic characteristics shown in Figs. 10 and 11 are very similar for all of the considered subsets and velocity options.

\section{Appendix B: CME motion}

The main problem included implicitly in the performed analysis is that Eq. (1) relates the instantaneous acceleration, a, with the instantaneous velocity, $v$, whereas the measurements provide only the mean acceleration $a_{\mathrm{m}}$ estimated over a wide distance range, $\Delta R$. Consequently, it would be erroneous to expect ad hoc that the measured mean acceleration is simply related to the mean speed, even if the drag is the dominant force in the considered distance range. Since this issue can cause a systematic error, we have to clarify its influence.

In Fig. 12a the drag-dominated $a(v)$-trajectory of one $a<0$ event is sketched in details. The velocity of CME at the beginning of measurement is denoted at the $x$-axis by " $\mathrm{b}$ " and at the end by "e". The mean velocity $v_{\mathrm{m}}$, defined in the $a=$ const. approximation as $v_{\mathrm{m}}=\left(v_{\mathrm{b}}+v_{\mathrm{e}}\right) / 2$, is marked by " $\mathrm{m}$ ". The mean acceleration, in the $a=$ const. approximation equivalent to $a_{\mathrm{m}}=\left(v_{\mathrm{e}}-v_{\mathrm{b}}\right) /\left(t_{\mathrm{e}}-t_{\mathrm{b}}\right)$, is depicted by the horizontal dashed line. If the solar wind speed and the parameter $\gamma_{2}$ are taken in the first approximation to be constant, the CME-trajectory (bold-dotted arrow) would follow the $a(v)$ line defined by $w_{\mathrm{b}}$ and $\gamma_{2 \mathrm{~b}}$. However, as the CME advances, the ambient solar wind speed increases whereas $\gamma_{2}$ decreases (Vršnak 2001; see also Sects. 4.2 and 5.2). Consequently, the CME
Table 5. Statistical characteristics of the n4v3-subset and (n6v3-subset).

\begin{tabular}{lccc}
\hline \hline Parameter & $\begin{array}{c}\text { Mean } \\
\text { value }\end{array}$ & $\begin{array}{c}\text { Standard } \\
\text { deviation }\end{array}$ & Median \\
\hline$R_{\mathrm{b}}$ & $3.4(3.3)$ & $1.0(0.9)$ & $3.1(3.1)$ \\
$R_{\mathrm{e}}$ & $14.0(15.2)$ & $6.9(6.8)$ & $12.5(14.0)$ \\
$R_{\mathrm{m}}$ & $8.7(9.3)$ & $3.6(3.5)$ & $8.0(8.6)$ \\
$\Delta R$ & $10.6(11.8)$ & $6.7(6.6)$ & $9.2(10.7)$ \\
$\phi$ & $73(77)$ & $73(74)$ & $53(57)$ \\
$v_{\mathrm{m}}\left(\mathrm{km} \mathrm{s}^{-1}\right)$ & $465(461)$ & $295(241)$ & $400(412)$ \\
$a\left(\mathrm{~m} \mathrm{~s}^{-2}\right)$ & $-1.2(-0.6)$ & $20.6(14.6)$ & $-0.2(0.0)$ \\
\hline
\end{tabular}

"slides" across $a(v)$-curves, ending at the one defined by $w_{\mathrm{e}}$ and $\gamma_{2 \mathrm{e}}$, as sketched by the bold arrow. Note that the line depicting the mean acceleration intersects the bold arrow at the velocity $v$ different from $v_{\mathrm{m}}$. Furthermore, note that the intercept of the $a_{\mathrm{m}}$ and $v_{\mathrm{m}}$ line is below both depicted $a(v)$-curves, i.e., it lies on a curve of too large $\gamma_{2}$, corresponding to some distance smaller than $R_{\mathrm{b}}$. On the other hand, the intercept of the $a_{\mathrm{m}}$ line with $v_{\mathrm{b}}$ is located between the "initial" and "final" $a(v)$-curve, i.e., it is associated with $\gamma_{2}$ corresponding to some distance between $R_{\mathrm{b}}$ and $R_{\mathrm{e}}$, presumably close to $R_{\mathrm{m}}$.

If we want to inspect quantitatively the effect sketched in Fig. 12a, it is instructive to consider firstly what mean acceleration would be measured if an object would move according to Eq. (1) in a homogeneous medium ( $w=$ const., $\gamma_{2}=$ const.; depicted by the bold-dashed arrow in Fig. 12a). The mean acceleration of the object, evaluated from the 2 nd order polynomial fit to the distance-time data can be expressed, by definition, as:

$a_{\mathrm{m}}=\frac{\left(v_{\mathrm{e}}-v_{\mathrm{b}}\right)}{\left(t_{\mathrm{e}}-t_{\mathrm{b}}\right)}=\frac{\left(\hat{v}_{\mathrm{e}}-\hat{v}_{\mathrm{b}}\right)}{\left(t_{\mathrm{e}}-t_{\mathrm{b}}\right)}$,

where $v_{\mathrm{b}}$ and $v_{\mathrm{e}}$ denote the rest-frame velocity at the beginning $\left(t_{\mathrm{b}}\right)$ and at the end $\left(t_{\mathrm{e}}\right)$ of the measurements, whereas $\hat{v}_{\mathrm{b}}$ and $\hat{v}_{\mathrm{e}}$ represent the corresponding velocities relative to the ambient gas ( $\hat{v}=v-w=v-$ const.).

The solution of Eq. (1) in the case $v_{\mathrm{b}}>w$ reads:

$\hat{v}_{\mathrm{e}}=\hat{v}_{\mathrm{b}} \mathrm{e}^{-\gamma_{2} \Delta \hat{r}}$,

where $\Delta \hat{r}=\hat{r}_{\mathrm{e}}-\hat{r}_{\mathrm{b}}$ is the distance traveled relative to the ambient gas in the time $\Delta t=t_{\mathrm{e}}-t_{\mathrm{b}}$. Integrating $\mathrm{d} \hat{r}=\hat{v} \mathrm{~d} t$ one finds another form of the solution:

$\hat{v}_{\mathrm{e}}=\frac{\hat{v}_{\mathrm{b}}}{1+\hat{v}_{\mathrm{b}} \gamma_{2} \Delta t}$

(see also Cargill et al. 1996), providing:

$\Delta t=\frac{\mathrm{e}^{\gamma_{2} \Delta \hat{r}}-1}{\gamma_{2} V_{\mathrm{b}}}$

Substituting Eqs. (9) and (7) into Eq. (6) one finds:

$a_{\mathrm{m}}=-\gamma_{2} \hat{v}_{\mathrm{b}} \hat{v}_{\mathrm{e}}=-\gamma_{2}\left(v_{\mathrm{b}}-w\right)\left(v_{\mathrm{e}}-w\right)$,

which is different from the ad hoc expected $\gamma_{2}\left(v_{\mathrm{m}}-w\right)\left(v_{\mathrm{m}}-w\right)$. Using Eq. (7) one can rewrite Eq. (10) as:

$a_{\mathrm{m}}=\gamma_{2}\left(v_{\mathrm{b}}-w\right)^{2} \mathrm{e}^{-\gamma_{2} \Delta \hat{r}}=\gamma_{2}^{*}\left(v_{\mathrm{b}}-w\right)^{2}$, 

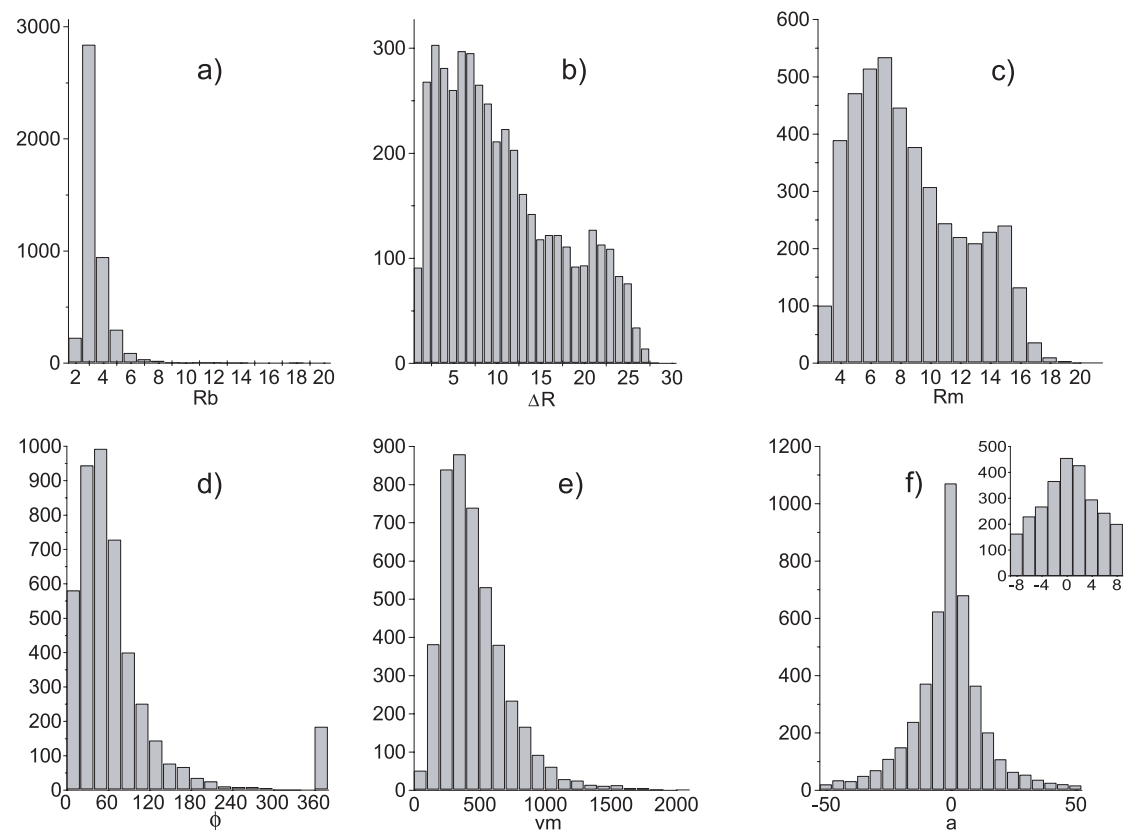

Fig. 10. Distribution of CME parameters in the $\mathrm{n} 4 \mathrm{v} 3$-subset: a) initial radial distances $R_{\mathrm{b}}$ (bins $\Delta R=1$ ); b) radial distance range $\Delta R=R_{\mathrm{e}}-R_{\mathrm{b}}$; c) mean radial distances $R_{\mathrm{m}}=\left(R_{\mathrm{e}}+R_{\mathrm{b}}\right) / 2$; d) angular widths $\phi$ (bins $\Delta \phi=20^{\circ}$ ); e) mean velocities $v_{\mathrm{m}}\left(\right.$ bins $\Delta v_{\mathrm{m}}=100 \mathrm{~km} \mathrm{~s}{ }^{-1}$ ); f) mean accelerations $a_{\mathrm{m}}$ (bins $\Delta a=5 \mathrm{~m} \mathrm{~s}^{-2}$ ); in the inset the central part of the distribution is presented (bins $\Delta a=2 \mathrm{~m} \mathrm{~s}^{-2}$ ).
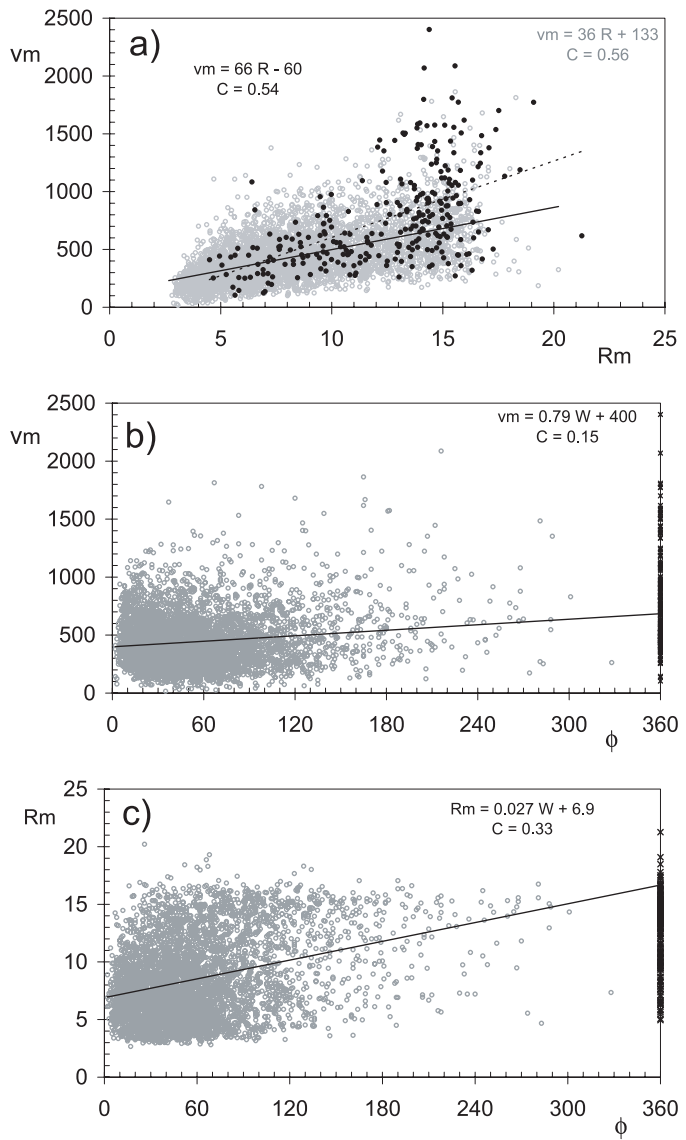

Fig. 11. Relation between different CME parameters in the n4v3-subset: a) $v_{\mathrm{m}}\left(R_{\mathrm{m}}\right)$ - black dots and the dashed linear least squares fit represent $\phi=360^{\circ}$ events. The $\phi<360^{\circ}$ events are depicted by gray circles and full-line least squares fit. b) $\left.v_{\mathrm{m}}(\phi) ; \mathbf{c}\right) R_{\mathrm{m}}(\phi)$. In b) and c) the $\phi=360^{\circ}$ events (crosses) are excluded from the fit. where we abbreviated $\gamma_{2}^{*}=\gamma_{2} \mathrm{e}^{-\gamma_{2} \Delta \hat{r}}$. So, if one would take the measured values $a_{\mathrm{m}}$ and $v_{\mathrm{b}}$ (or equivalently $v_{\mathrm{b}} \approx v_{3}$ as done in Sect. 4) to estimate $\gamma_{2}^{*}$ utilizing Eq. (11), its value would be smaller than the real value $\gamma_{2}$ by the factor $X=\mathrm{e}^{-\gamma_{2} \Delta \hat{r}}$. Using Eq. (8), the deviation $X$ can be expressed as:

$X=\left(\gamma_{2} \hat{v}_{\mathrm{b}} \Delta t+1\right)^{-1}$.

For a given CME the deviation $X$ depends on the initial velocity and the time interval during which the kinematics was measured, and this varies from one event to another.

Actually, if $\Delta t$ increases, $R_{\mathrm{m}}=R_{\mathrm{b}}+\Delta R / 2$ increases too, which means that $\gamma_{2}$ decreases (Sect. 4 ). Thus, the two effects partly compensate each other. In Fig. $12 \mathrm{~b}$ we show the deviation $X$ as a function of $R_{\mathrm{m}}$. In evaluating $X\left(R_{\mathrm{m}}\right)$ we have used $w=w\left(R_{\mathrm{m}}\right)$ as given by Eq. (3), and $\gamma_{2}=\gamma_{2}\left(R_{\mathrm{m}}\right)$ estimated utilizing the two scalings displayed in the bottom panel of Fig. $4 \mathrm{~b}$ $\left(\gamma_{2}=5.9 \times 10^{-6} R^{-2}\right.$ and $\left.\gamma_{2}=0.76 \times 10^{-6} R^{-0.98}\right)$.

Note that in the considered approximation, in most of events ( $4<R_{\mathrm{m}}<15$; see Appendix A) the value of $\gamma_{2}^{*}$ is underestimated for not more than $50 \%$ with respect to $\gamma_{2}$.

Let us now consider the more realistic situation, where the wind speed and density, as well as the dimensions and density of CME, depend on the radial distance, so $w$ and $\gamma_{2}$ are functions of $R$. We presume that $w(R)$ increases, whereas $\gamma_{2}(R)$ decreases.

We performed a series of numerical simulations from which we estimated $a_{\mathrm{m}}$ to determine how much the approximate value $\gamma_{2}^{*}\left(R_{\mathrm{m}}\right)=a_{\mathrm{m}} /\left(v_{\mathrm{b}}-w\left(R_{\mathrm{m}}\right)\right)^{2}$ differs from the true $\gamma_{2}\left(R_{\mathrm{m}}\right)$. Again we used the two $\gamma_{2}(R)$ scalings from the bottom panel of Fig. $4 \mathrm{~b}$, combined with the solar wind model given by Eq. (3). The results are illustrated by Fig. 12c, revealing a complex situation. The deviation $X$ is generally $X<1$, but it can also be $X>1$, i.e., $\gamma_{2}^{*}$ can be overestimated (see also the 
B. Vršnak et al.: Dynamics of CMEs, Online Material p 4
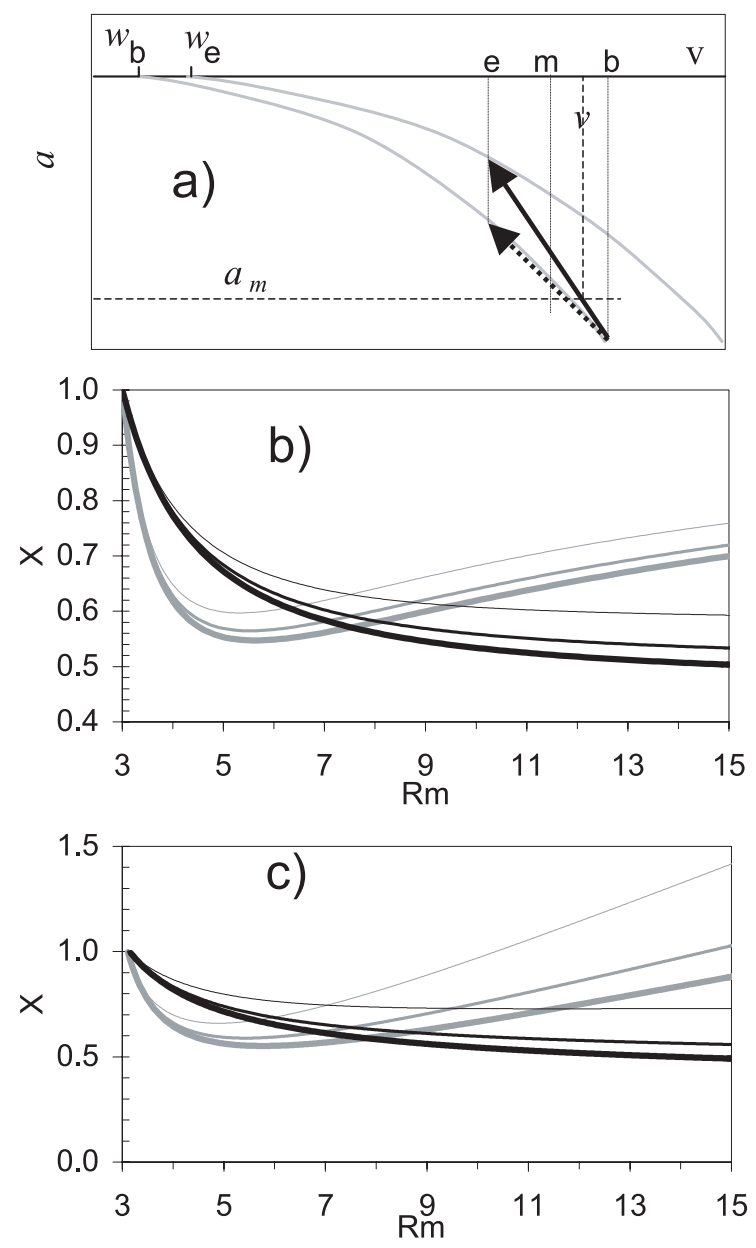

Fig. 12. a) Comparison of the $a(v)$-trajectory of an event moving in the $w=$ const. environment (bold-dotted arrow) with the one moving in the solar wind where the density decreases and flow speed $w$ increases (bold arrow). The initial, mean and final velocities are denoted by $b$, $\mathrm{m}$, and e, respectively. b) The deviation $X$ for the approximation $\gamma_{2}=$ const., $w=$ const. c) The deviation $X$ for the solar wind speed given by Eq. (3) combined with $\gamma_{2}=0.76 \times 10^{-6} R^{-0.98}$ (black) and $\gamma_{2}=5.9 \times$ $10^{-6} R^{-2}$ (gray). The successively thicker lines in b) and c) represent $v_{3}=1000, v_{3}=1500$, and $v_{3}=2000 \mathrm{~km} \mathrm{~s}^{-1}$.

explanation of Fig. 12a). The later case is more pronounced for larger values of the exponent $\beta_{R}$ in the $\gamma(R) \propto R^{-\beta_{R}}$ scaling. When $v_{\mathrm{m}}$ is considered instead of $v_{\mathrm{b}}$, the value of $\gamma_{2}^{*}$ is always overestimated. For this reason in the previous analysis (Sects. 3 and 4) we relied more on the velocity $v_{3}$ than on $v_{\mathrm{m}}$. Note that weak-acceleration events are not affected much by the choice of $v_{3}$ or $v_{\mathrm{m}}$.

Finally, we stress another important aspect of employing the velocity $v_{3}$. Considering what happens in the $a-v$ graph

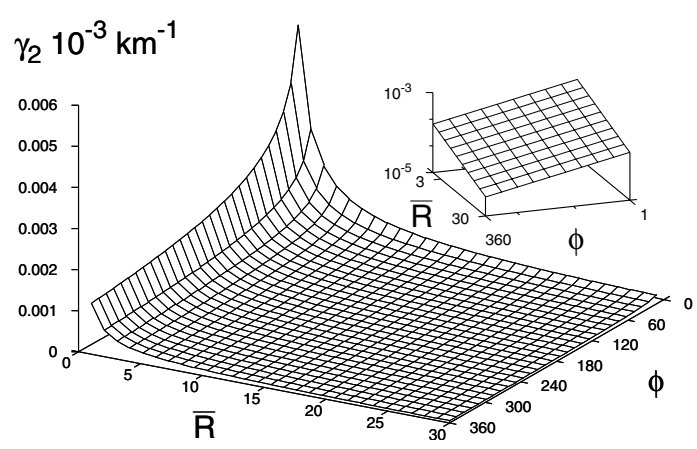

Fig. 13. The fitted $\gamma_{2}(R, \phi)$ surface obtained by using Eq. (13). In the inset log-scale presentation is shown.

when the $a_{\mathrm{m}}\left(v_{\mathrm{m}}\right)$ data are replaced by $a_{\mathrm{m}}\left(v_{3}\right)$, one finds that the usage of $v_{3}$ shifts the $a_{\mathrm{m}}>0$ events to lower velocities (to the left in the graph), and $a_{\mathrm{m}}<0$ events to higher velocities (to the right). This would tend to decrease the slope of the $a-v$ correlation if only the drag force dominated events would be present (see the explanation of Fig. 12a). However, a compensating effect appears at large velocities where we find also the events with large $a_{\mathrm{m}}>0$ (presumably those whose motion is still governed by the propelling force). These events, which tend to smear the correlation and decrease $\gamma_{1,2}$, are shifted to the left towards $v_{0}$, which reduces their influence in the $a(v)$ fitting. This is a reason reason why the $a_{\mathrm{m}}\left(v_{3}\right)$ relationship shows higher correlation coefficients than $a_{\mathrm{m}}\left(v_{\mathrm{m}}\right)$ relationship and a steeper slope $\gamma_{1}$.

In conclusion, let us note that Eq. (10) provides another possibility to estimate $\gamma_{2}(\phi, R)$ scaling, different from that performed in Sect. 4. Combining the observed values $a_{\mathrm{m}}, v_{\mathrm{b}}$, and $v_{\mathrm{e}}$, with the model values for the solar wind speed $w\left(R_{\mathrm{b}}\right)$ and $w\left(R_{\mathrm{e}}\right)$ as given by Eq. (3), one can estimate:

$\tilde{\gamma}_{2}=-\frac{a_{\mathrm{m}}}{\left(v_{\mathrm{b}}-w_{\mathrm{b}}\right)\left|\left(v_{\mathrm{e}}-w_{\mathrm{e}}\right)\right|}$

for each event. Here we meet a problem if the $v_{\mathrm{b}} \approx w_{\mathrm{b}}$ events are included - because of the small value in the denominator the outcome becomes highly unreliable. So we excluded the $\left|v_{\mathrm{b}}-w_{\mathrm{b}}\right|<50 \mathrm{~km} \mathrm{~s}^{-1}$ events. The remaining data are then fitted in the $\tilde{\gamma}_{2}(R, \phi)$ space using the function $\tilde{\gamma}_{2}(R, \phi)=\alpha \phi^{-\beta_{\phi}} R^{-\beta_{R}}$ (the plane in log-log-log presentation). The outcome is shown in Fig. 13: the parameters obtained by the linear least squares fit read $\alpha=0.015 \times 10^{-3} \mathrm{~km}^{-1}, \beta_{\phi}=0.42$, and $\beta_{R}=0.95$. So, the value of $\tilde{\gamma}_{2}$ decreases with $R$ and $\phi$. Note that the slopes $\beta_{\phi}$ and $\beta_{R}$ are consistent with those shown in Figs. $4 \mathrm{a}$ and $4 \mathrm{~b}$. 